\title{
Comunidades vegetales de las transiciones terrestre-acuáticas del páramo de Chingaza, Colombia
}

\author{
Udo Schmidt-Mumm ${ }^{1} \&$ Orlando Vargas Ríos ${ }^{2}$ \\ 1. Department of Limnology-Hydrobotany, University of Vienna, Althanstrasse 14, A-1091 Vienna, Austria; udo. \\ schmidt-mumm@univie.ac.at \\ 2. Departamento de Biología, Edificio 421, Facultad de Ciencias, Universidad Nacional de Colombia, Bogotá, \\ Colombia; jovargasr@unal.edu.co
}

Recibido 04-II-2011. Corregido 10-V-2011. Aceptado 08-VI-2011.

\begin{abstract}
Plant communities in the terrestrial-aquatic transition zone in the paramo of Chingaza, Colombia. High Andean paramo ecosystems are an important water resource for many towns, and major cities in this region. The aquatic and wetland vegetation of different paramo lakes, pond, swamps and bogs was studied according to the classical phytosociological approach, which is based on homogenous stands, but excludes any border phenomena or transitional zone. The present research aimed at determining the aquatic and wetland vegetation along different moisture gradients. A total of 89 species in 30 transects were reported, of which Crassula venezuelensis, Carex bonplandii, Callitriche nubigena, Eleocharis macrostachya, Ranunculus flagelliformis, $R$. nubigenus, Eleocharis stenocarpa, Galium ascendens y Alopecurus aequalis were present in more than one third of the transects. Numerical classification and indicator species analysis resulted in the definition of the next 18 communities: 1) Calamagrostis effusa, 2) Sphagnum cuspidatum, 3) Cyperus rufus, 4) Eleocharis stenocarpa, 5) Carex acutata, 6) Pоа апnиа, 7) Valeriana sp., 8) Ranunculus flagelliformis, 9) Carex bonplandii, 10) Festuca andicola, 11) Muhlenbergia fastigiata, 12) Elatine paramoana, 13) Isoëtes palmeri, 14) Crassula venezuelensis, 15) Lilaeopsis macloviana, 16) Callitriche nubigena, 17) Potamogeton paramoanus and 18) Potamogeton illinoensis. The ordination of communities reveals the presence of three different aquatic-terrestrial gradients which are related to the life form structure of species that characterized the various communities. We concluded that patchiness and heterogeneity of the vegetation is mainly the result of alterations caused by human activities (burning, cattle raise and material extraction for road and dam construction). Rev. Biol. Trop. 60 (1): 35-64. Epub 2012 March 01.
\end{abstract}

Key words: Andes, aquatic communities, macrophytes, mire vegetation, paramo, terrestrial-aquatic gradient, zonation.

Los páramos son biomas exclusivos de las montañas neotropicales, localizados aproximadamente entre los 3100 y 4700 m.s.n.m., con presencia en los sistemas andinos de Venezuela, Colombia y Ecuador, y con extensiones en Costa Rica y Panamá (Rangel 2000a). La flora vascular de las regiones tropicales de alta montaña de Centro y Suramérica es considerada una de las más ricas en géneros y especies del mundo (Cleef 1981, 2008). En un intento por cuantificar la riqueza florística de los páramos, Luteyn (1999) registró 447 géneros y alrededor de 3045 especies y subespecies de espermatofitos para la región. Sin embargo, con la adición del listado de la flora actualmente conocida para los páramos y la región del bosque altoandino de Colombia, Rangel (2000d) logra ampliar el número de géneros y especies a un total de 644 y 4696 respectivamente.

Los páramos son extremadamente vulnerables a los efectos de la actividad humana, debido a que la vegetación apenas tolera bajas frecuencias de quema y pastoreo (Hofstede 1995, Verweij 1995). Como práctica 
generalizada de pastoreo el ganado se deja libre y sin rotación en páramos cercanos a los asentamientos y generalmente los animales terminan agrupándose alrededor de las haciendas, cerca de fuentes de agua, o áreas con forrajes accesibles producto de la quema. La quema de pajonales para el posterior pastoreo se volvió una práctica común en los páramos húmedos, y actualmente es la actividad que afecta grandes extensiones. Por otro lado, la diversidad de las comunidades de plantas también es afectada por el pastoreo debido a los impactos directos por herbivoría y el pisoteo permanentes, que alteran el balance competitivo entre las especies los cuales cambian las oportunidades para el establecimiento de nuevas plantas (Verweij \& Budde 1992, Verweij \& Kok 1992, Hofstede et al. 1995, Verweij 1995, Vargas 1996, Molinillo \& Monasterio 2002, Vargas et al. 2002). En general los estudios concluyen que la composición de la vegetación, la estructura y los bancos de semilla se ven fuertemente afectados por el fuego y el pastoreo (Hofstede 1995, Cardenas et al. 2002, Premauer \& Vargas 2004, Cárdenas \& Vargas 2008).

Aunque, frecuentemente se resalta la importancia de los cuerpos de agua de la alta montaña como recurso hídrico. Los estudios realizados en la vegetación acuática son pocos, la mayoría de han sido efectuados en diferentes regiones paramunas de Colombia. Venezuela Cuello \& Cleef (2009) describieron recientemente seis asociaciones en un estudio de corte fitosociológico realizado en lagunas y turberas del Páramo de Guaramacal y El Pumar. Entre los trabajos pioneros que tratan sobre la vegetación acuática y de pantanos de los páramos en los alrededores de la Sabana de Bogotá figuran los de Cleef (1981), quien reconoce y clasifica 42 asociaciones/comunidades acuáticas y de pantanos para la Cordillera Oriental de Colombia; según la posición orográfica y humedad atmosférica, el anterior autor también diferencia los páramos húmedos con presencia de bambú de aquellos dominados por pajonales o pastizales y clima seco. En un estudio de la vegetación del páramo de Monserrate Vargas \& Zuluaga (1985) reconocen únicamente dos asociaciones que caracterizan lugares pantanosos a turbosos. Las características físicoquímicas y la composición florística de la brioflora de diferentes depósitos turbosos en los páramos circundantes a la ciudad de Bogotá son reseñadas por Sánchez et al. (1989). Éstos autores también presentan un modelo hipotético de sucesión y resaltan la importancia que juega la brioflora en del proceso de terrización de dichos depósitos. Posteriormente Sánchez \& Rangel (1990) proponen el arreglo sintaxonómico para la vegetación anteriormente estudiada, la cual comprende cuatro alianzas y 11 asociaciones. La vegetación azonal del páramo de Sumapaz fue recientemente retomada y revisada por Cleef et al. (2008), quienes reclasifican, modifican y corrigen varias de las sintaxa anteriormente descrita por Cleef (1981). En un intento por organizar las diferentes comunidades actualmente descritas para los páramos de Colombia, Rangel (2000c) documenta la presencia de 327 comunidades o tipos de vegetación. Cuatro de estos corresponden a una vegetación de turberas, dos a una vegetación de cojines y 46 a la vegetación acuática o de pantanos.

El Parque Nacional Natural Chingaza (PNN Chingaza), es parte del complejo de páramos de Chingaza, fue creado principalmente con el objeto de conservar y administrar un ecosistema, que brinda alrededor del $80 \%$ del agua potable de la ciudad de Bogotá y de algunos municipios aledaños. Con excepción del embalse de Chuza y la laguna de Chingaza, las lagunas del Parque son generalmente de tamaño pequeño con áreas que van de los 0.06ha a los 8ha y profundidades máximas que no sobrepasan los 8m (Gaviria 1993). Las aguas son por lo general oligotróficas, con bajas concentraciones en nutrientes y bajos valores de conductividad, alcalinidad y dureza. Aparentemente el nitrógeno resulta ser de manera general el factor limitante (Donato 1991, 2001, Donato et al. 1996). A pesar de la gran importancia del PNN Chingaza como recurso hídrico, los trabajos realizados en la vegetación acuática y semiacuática del parque son escasos. Algunos levantamientos de la 
vegetación fueron realizados inicialmente por Cleef (1981) en el páramo de Palacio y luego ampliados por Franco et al. (1986) a los alrededores de la laguna de Chingaza. Posteriormente Rangel \& Ariza (2000) reevalúan y complementan el anterior trabajo.

En términos generales todos los trabajos realizados sobre la vegetación acuática y de pantanos de los páramos siguen el corte fitosociológico-sintaxonómico desarrollado por Braun-Blanquet (1979), método que se basa en muestreos de áreas homogéneas y excluye de todo tipo de fenómenos de borde o transicionales (ecotonos). Debido a que la mayoría de los estudios se han concentrado en las áreas de vegetación homogénea, poco se sabe acerca de los patrones de la riqueza de especies y de la composición de las comunidades a lo largo de las zonas transicionales. Sin embargo, el estudio de estas zonas de transición no solo juega un papel importante en la definición teórica de la estructura de las comunidades y los patrones de zonación (Shipley \& Keddy 1987, Hoagland \& Collins 1997, Keddy 2000), sino también en los aspectos prácticos relacionados con la determinación y delimitación de los márgenes o bordes de los humedales los cuales son requisitos importantes en el manejo y conservación de los mismos (Carter et al. 1994, Davis et al. 1996, Holland 1996, Kirkman et al. 1998). El propósito del presente estudio fue el análisis de 1) la estructura florística, biotipológica y cuantitativa de la vegetación en los diferentes gradientes hídricos de los cuerpos de agua del PNN Chingaza, 2) el reconocimiento de grupos de especies que permitan definir cada una de las zonas del gradiente hídrico, y 3 ) la descripción de los tipos de vegetación que caracterizan la zonación de los diferentes cuerpos de agua.

\section{MATERIALES Y MÉTODOS}

Área de estudio: El PNN Chingaza, localizado al suroriente de Bogotá entre los $4^{\circ} 51^{\prime}-4^{\circ} 20^{\prime} \mathrm{N}$ y $73^{\circ} 30^{\prime}-73^{\circ} 55^{\prime} \mathrm{W}$, comprende alrededor de las 76600 hectáreas con rangos latitudinales que van desde los 800 hasta los 4 020m.s.n.m. (Fig. 1). Las temperaturas presentan un amplio rango que va desde los $6^{\circ} \mathrm{C}$ en la cima de la serranía de los Farallones hasta los $21^{\circ} \mathrm{C}$ en la cota de los 800 m.s.n.m. Las temperaturas máximas medias oscilan entre los 12 y $14^{\circ} \mathrm{C}$ y las mínimas medias llegan a valores de $-2^{\circ} \mathrm{C}$. El régimen de lluvias es de tipo unimodal biestacional, con las mayores precipitaciones distribuidas entre abril y finales de septiembre (máximas en junio) y las menores entre octubre y finales de marzo (mínimas en febrero). Una descripción más detallada sobre el ambiente físico del PNN Chingaza se puede encontrar en Vargas \& Pedraza (2004). El estudio de la vegetación acuática y semiacuática en el PNN Chingaza se realizó en tres sectores caracterizados por diferencias climáticas e hidrológicas.

Sector I: Corresponde a la zona norte del parque y abarca los cuerpos de agua localizados entre los 3200 y 3 600m.s.n.m. en los alrededores de las lagunas de Buitrago y el sector conocido como Piedrasgordas. Representa un páramo semihúmedo con precipitaciones medias anuales de $1200 \mathrm{~mm}$.

Sector II: Corresponde al plano aluvial del río La Playa y Valle de los Frailejones entre alturas de 3100 y 3 300m.s.n.m. Se encuentra caracterizado por un páramo húmedo y recibe en promedio $2800 \mathrm{~mm}$ de precipitación al año.

Sector III: Abarca el plano aluvial del río Frío y la laguna de Chingaza con alturas alrededor de los 3250 m.s.n.m. Este sector también se encuentra representado por características de páramo húmedo y recibe en promedio $2126 \mathrm{~mm}$ de precipitación al año.

Muestreo: Con el fin de adelantar el reconocimiento de la flora acuática y palustre del PNN Chingaza se realizaron una serie de colecciones de herbario, conjuntamente con el inventario de los transectos. Las colecciones siguen la numeración del primer autor y se encuentran depositadas en el herbario de la Universidad Javeriana (HPUJ) y en el Herbario Nacional Colombiano (COL). La identificación del material botánico se realizó con ayuda de claves y descripciones taxonómicas, la cual se complementó en algunos casos mediante la 


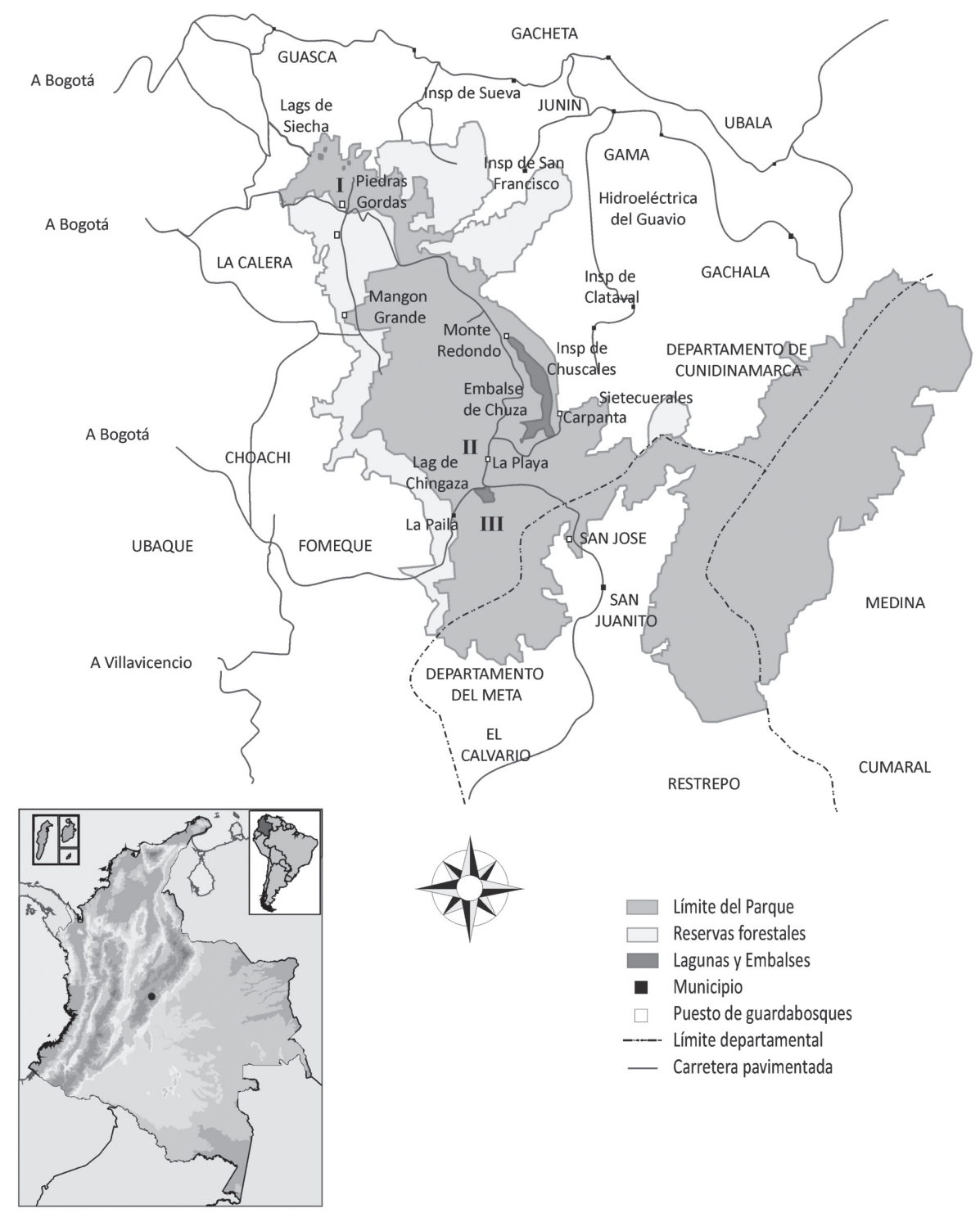

Fig. 1. Ubicación del Parque Nacional Natural Chingaza y áreas de muestreo. I = Sector Piedras Gordas-Buitrago, II = Plano aluvial del Río la Playa, III = Plano aluvial del Río Frío-Laguna Chingaza (adaptado de Vargas \& Pedraza 2004).

Fig. 1. Map of the Chingaza National Natural Park and study sites. I = Piedras Gordas-Buitrago sector, II = Alluvial plane of the La Playa river, III = Alluvial plane of the river Río Frío-Chingaza lake (adapted from Vargas \& Pedraza 2004).

comparación de material de herbario (COL) y la asesoría de botánicos especializados.

El estudio de la vegetación en los diferentes cuerpos de agua del Parque se realizó en los meses de septiembre a noviembre de 1988 (siete transectos, época húmeda), junio a septiembre (16 transectos, época húmeda) y diciembre (cuatro transectos, época seca) de 1989, y enero (tres transectos, época seca) de 1990. El tipo de cuerpos de agua estudiados corresponde a turberas, pantanos, charcas o cubetas y lagunas. El levantamiento de la 
vegetación se efectúo mediante transectos en banda orientados a lo largo del gradiente terrestre-acuático. Los transectos median entre $10 \mathrm{y}$ $20 \mathrm{~m}$ de longitud según al ancho de la transición terrestre-acuático ocupada por vegetación. Uno de los extremos de los transectos se ubicó sobre la vegetación terrestre, mientras que el extremo acuático quedaba definido por la ausencia de la vegetación sumergida (sedimento descubierto). Con excepción de la Laguna de Chingaza, y dado el grado de terrización de los cuerpos de agua, la profundidad máxima con vegetación sumergida no sobrepasaba los $50 \mathrm{~cm}$. Cada transecto se dividió sistemáticamente en unidades de muestreo de $50 \times 50 \mathrm{~cm}\left(0.25 \mathrm{~m}^{2}\right)$. Para determinar la abundancia de las especies como frecuencia de enraizamiento local se dividió nuevamente cada cuadrado de muestreo en cinco sub-cuadrados de $10 \times 50 \mathrm{~cm}$ orientados perpendicularmente al gradiente acuático. De esta forma se le asigna a cada especie en determinado cuadrado de muestreo un valor entre cero (ausente en todos los sub-cuadrados) y cinco (presente en los cinco sub-cuadrados).

La estructura biotipológica de la vegetación acuática y semiacuática del Parque se analizó a partir de la clasificación de las plantas en formas de vida (biotipos). De manera general estas se definieron de acuerdo a la clasificación tradicional como plantas enraizadas sumergidas, plantas enraizadas de hojas flotantes, plantas errantes y plantas enraizadas emergentes o helófitos (Sculthorpe 1967, Hutchinson 1975). Dado que muchas especies son representadas por la forma enraizada emergente se consideró de utilidad subdividir esta categoría en helófitos herbáceos, helófitos graminoides y helófitos junciformia o juncoides (Danserau 1959, Hutchinson 1975). El grupo de plantas que crecen en las zonas litorales sobre suelos húmedos, frecuentemente también denominadas como plantas higrófilas, se definieron simplemente como terrestres.

La evaluación de las variaciones florísticas y cuantitativas a lo largo del gradiente hídrico se realizó con un enfoque exploratorio mediante diferentes técnicas de clasificación y ordenación. En la búsqueda de grupos de especies que puedan definir las sucesivas zonas sobre el gradiente acuático-terrestre se parte de la idea de una zonación bien definida, es decir caracterizada por comunidades herbáceas de estructura biotipológica y florística distinta que se reemplazan unas a otras a lo largo del gradiente hídrico (sumergidas o errantes -hojas flotantesenraizadas emergentes). Un enfoque similar para el estudio de las comunidades vegetales en transiciones terrestre-acuáticas es presentado por Jensén \& van der Maarel (1980). Con el fin de reducir el número de cuadrados de cada transecto en grupos florísticamente más homogéneos que expresan la zonación se seleccionó la clasificación TWINSPAN (“Análisis Doble-Entrada de Especies Indicadoras”). La base de esta técnicas politéticas divisivas es la ordenación. Originalmente descrito como un "análisis de especies indicadoras" (Hill et al. 1975) fue posteriormente renombrado a "análisis de ordenación dicotomizado" (Hill 1979) con el argumento de que la base de la técnica es la división de ordenaciones y no la selección de especies indicadoras. Sin embargo, al igual que otras técnicas divisivas, la clasificación TWINSPAN presenta varias limitaciones entre las cuales figuran la imposibilidad de corregir la posición de las unidades mal clasificadas por una selección errónea aleatoria de alguna de las especie preferencial, la influencia de especies raras que se encuentran además en unidades de muestreo con pocas especies (Kautsky \& van der Maarel 1990), y sobre todo la dificultad de manejar más de un gradiente ambiental importante (McCune \& Grace 2002).

El producto de la clasificación de cada transecto, considerado aquí como una población o agrupación local que refleja parte de la zonación del gradiente de inundación, nuevamente se organizó en una matriz de datos representada por el total de especies de todos los transectos muestreados y el total de agrupaciones derivados por la clasificación TWINSPAN. En este caso los valores cuantitativos se encuentran representados por la suma de los valores de presencia de cada especie en los diferentes cuadrados agrupados, los cuales se expresaron como frecuencias porcentuales. 
Por ejemplo: si un grupo derivado de la clasificación TWINSPAN contiene seis cuadrados el número total de sub-cuadrados será $6 \mathrm{x}$ $5=30$ sub-cuadrados. Ahora, si la especie A se presenta en todos los 30 sub-cuadrados su frecuencia porcentual será (30/30)100 $=100 \%$; si aparece en 13 sub-cuadrados su frecuencia será $(13 / 30) 100=43 \%$. Estos valores se agruparon en una nueva matriz primaria la cual fue el punto de partida de la posterior clasificación. De los subsiguientes análisis se excluyeron las especies que se presentaron en menos del 2\% de las agrupaciones. Por lo general muchas de estas especies corresponden a la vegetación terrestre, al igual que pantanos y turberas arbustivas, cuya distribución óptima se encuentra representada fuera de los límites del gradiente analizado en el presente trabajo.

La clasificación de la matriz primaria (frecuencias porcentuales de las especies en los grupos) se realizó mediante el índice de Bray-Curtis y aglomeración por ligamiento flexible con un valor de $\beta=-0.25$. La división del dendrograma en tipos de comunidades que muestran el mayor nivel de información se definió cuantitativamente mediante el análisis de especies indicadoras (Indicator Species Analysis, ISA). Como nivel de corte del dendrograma se uso el menor valor de las probabilidades medias calculado durante el análisis ISA (Dufrêne \& Legendre 1997, Legendre \& Legendre 1998). De igual manera se determinaron las especies indicadoras para cada comunidad resultantes de la anterior clasificación. Dado que el número de especies varía de acuerdo al área de muestreo para cada grupo o comunidad, la riqueza de especies por grupo TWINSPAN se estimo mediante la expresión: riqueza $=$ número de especie $/ \log _{10}$ área del grupo inventariado (Luken \& Bezold 2000). El área se considero en $\mathrm{cm}^{2}$ para evitar una división por cero $\left(1 \mathrm{~m}^{2}=10000 \mathrm{~cm}^{2}\right)$.El promedio de los valores de riqueza de los grupos incluidos en cada comunidad define la riqueza específica media de la comunidad. Diferencias entre las riquezas medias de las diferentes comunidades se determinaron mediante el análisis de varianza no paramétrico Krukal-Wallis.
Las comparaciones múltiples por pares se realizaron con el procedimiento de Dunn y ajuste de Bonferroni.

El análisis ISA calcula los valores indicadores de las especies mediante la multiplicación de la abundancia relativa de cada especie en un grupo específico por la frecuencia relativa de la ocurrencia de la especies en ese grupo. La significancia estadística $(\mathrm{p} \leq 0.05)$ de los valores indicadores se determinó mediante la prueba de simulación Monte Carlo con 1000 aleatorizaciones (McCune \& Grace 2002).El porcentaje de la frecuencia relativa y el nivel de significancia generada por la prueba de simulación para cada especie indica la probabilidad de encontrar una especie en dicha comunidad.

Con el fin de explorar la estructura florística y cuantitativa de las comunidades obtenidas mediante el análisis de agrupamiento, al igual que su relación con el gradiente hídrico, se siguió la estrategia de ordenación conocida como "Escalamiento Multidimensional no Métrico" (Non-Metric Multidimensional Scaling, NMDS). Esta parte de una matriz de distancias Bray-Curtis. La cantidad de dimensiones requeridas para la ordenación final se determinó mediante el procedimiento lento y completo del modo "autopiloto" explicado en McCune \& Grace (2002): Número máximo de iteraciones $=500$, criterio de inestabilidad $=0.0000001$, número de ejes iniciales $=6$, número de recorridos reales $=250$, número de recorridos aleatorizados $=250$. Los ejes de la ordenación final se orientaron mediante rotación "varimax". La proporción de la varianza representada por los ejes de ordenación se determino mediante correlaciones Post-hoc entre las distancias del espacio de ordenación y el espacio original (McCune \& Grace 2002). La tendencia de las formas de vida sobre el gradiente hídrico se expresó mediante vectores en un gráfico sobrepuesto al plano de ordenación NMDS (Biplot). Todos los análisis multivariados (TWINSPAN, Aglomeración Flexible, ISA, NMDS) se realizaron mediante el programa PC-ORD v. 5.18 (McCune \& Mefford 2006), y la prueba Kruskal-Wallis mediante XLSTAT-Pro (Addinsoft 2008). 


\section{RESULTADOS}

Florística: Se registró un total de 89 especies en 30 transectos, de las cuales una era Charophyta, 10 Bryophyta, cinco Pteridophyta, 31 monocotiledóneas y 42 eucotiledóneas. La lista taxonómica completa se presenta con algunas anotaciones en el Apéndice. El mayor número de taxones se presentó en las familias Poaceae, Cyperaceae, Scrophulariaceae y Asteraceae. Especies presentes en más de un tercio de los transectos fueron Crassula venezuelensis (60\%), Carex bonplandii (50\%), Callitriche nubigena (50\%), Eleocharis macrostachya (46.7\%), Ranunculus flagelliformis (46.7\%), Ranunculus nubigenus (40\%), Eleocharis stenocarpa (36.7\%), Galium ascendens (33.3\%) y Alopecurus aequalis (33.3\%).

Comunidades: La clasificación TWINSPAN de cada transecto resultó en un total de 147 grupos. 17 especies se presentaron en menos de tres grupos (2\%) y se excluyeron de los análisis numéricos subsiguientes (Apéndice). El mayor número de especies son representadas por plantas terrestres (33.8\%), seguido por las formas de vida de los tipos helófitos graminoides $(18.3 \%)$ y herbáceos (18.3\%), helófitos juncoides (8.8\%), enraizadas sumergidas $(8.8 \%)$, helófitos briófitos $(7 \%)$, enraizadas con hojas flotantes $(4.2 \%)$ y por último las plantas errantes (1.4\%) (Fig. 2).

Mediante el análisis ISA del dendrograma por agrupamiento flexible se reconocieron 61 especies indicadoras en 18 comunidades (Fig. 3). $10(33 \%)$ de los transectos son representados por una comunidad, 14 (47\%) por dos comunidades, cinco (17\%) por tres comunidades, y un (3\%) transecto por cinco comunidades. De otro lado seis comunidades (33\%) comprenden el $66 \%$ de las especies indicadoras. El mayor número de especies indicadoras se presentaron en las comunidades C-6, C-1, C-9 y C-7 (Cuadro 1). La ordenación NMDS de las comunidades presenta una solución tridimensional relativamente estable, con un estrés final moderado de 10.95, y una inestabilidad final de $<0.000001$. La proporción de la variación representada por los ejes de ordenación fue del $82 \%$, comprendida por el $20 \%$ en el eje uno, $25 \%$ en el eje dos y $37 \%$ en el eje tres. Las tendencias de la estructura biotipológica sobre el gradiente hídrico se encuentra representa en el primer eje por los helófitos graminoides y herbáceos, y las formas enraizadas sumergidas. El segundo eje define un gradiente representado por la vegetación terrestre y aquellas plantas enraizadas de hojas flotantes (Fig. 4). El tercer eje caracteriza diferencias entre las comunidades de lugares pantanosos con representantes

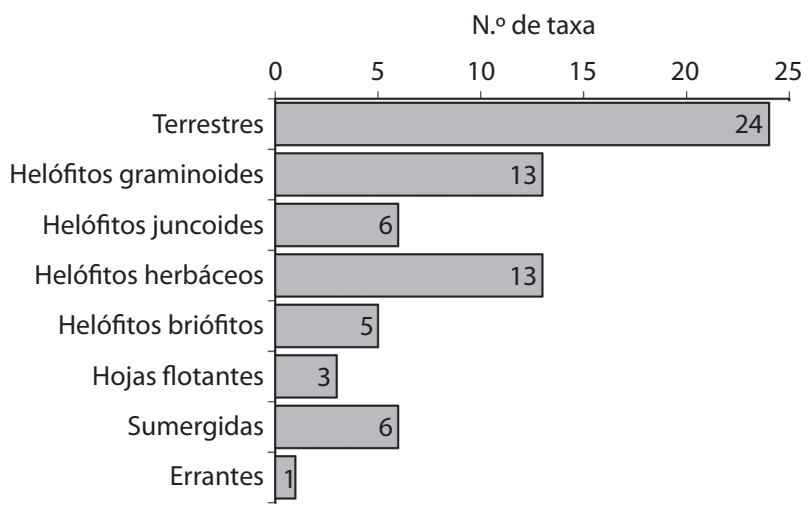

Fig. 2. Espectro biotipológico de los macrófitos acuáticos y semiacuáticos del PNN Chingaza.

Fig. 2. Life form spectrum of aquatic and wetland macrophytes in the Chingaza Park. 


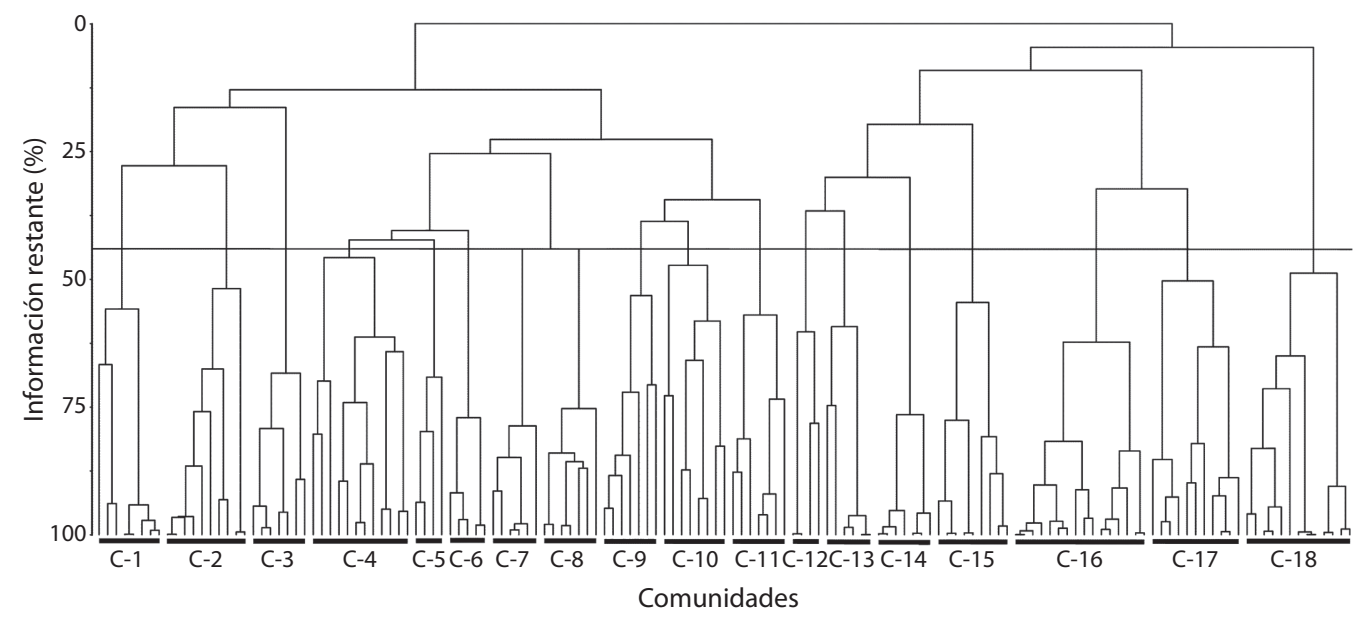

Fig. 3. Aglomeración flexible $(\beta=-0.25)$ de los grupos de cada transecto obtenidos mediante el análisis TWINSPAN. La línea horizontal define las comunidades representadas por el menor valor medio de las probabilidades de cada nivel de ligamiento calculado a través del análisis de especies indicadoras (ISA).

Fig. 3. Flexible clustering $(\beta=-0.25$ ) of the groups of each transect obtained by TWINSPAN analysis. The horizontal line defines the communities represented by the lowest mean values of probabilities for each of the clustering levels calculated in the indicator species analysis (ISA).

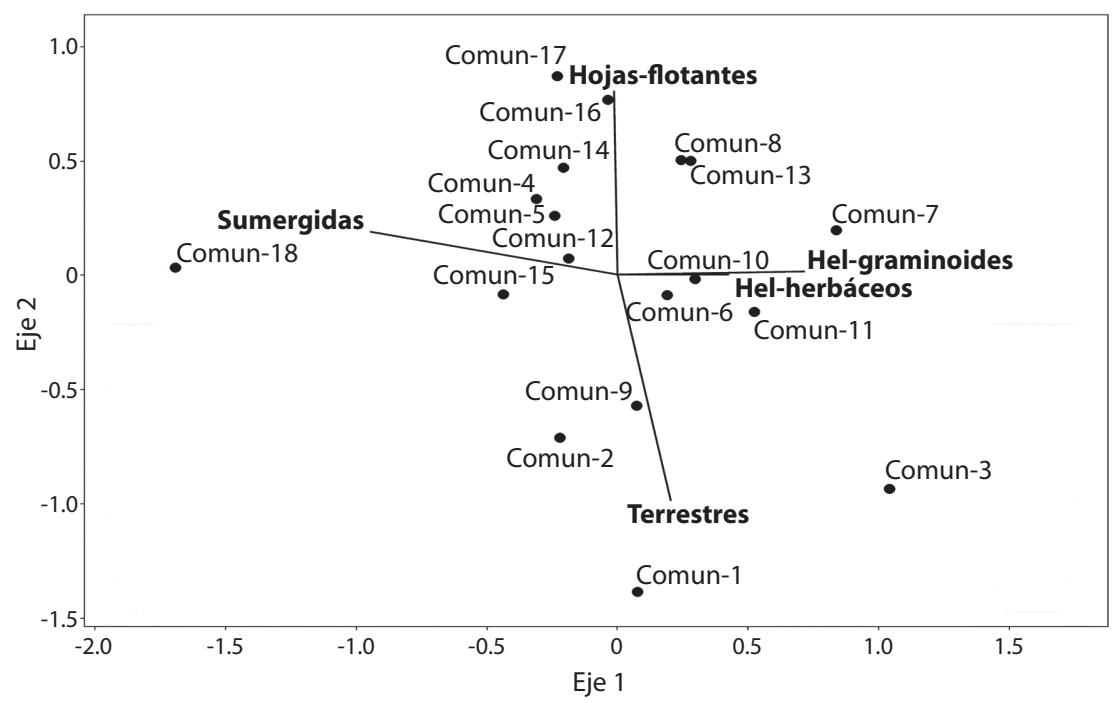

Fig. 4. Ordenación NMDS de las comunidades del PNN Chingaza en el primer y segundo eje. La sobreposición de las formas de vida señala tendencias en la estructura de la vegetación sobre el gradiente hídrico.

Fig. 4. NMDS ordination of communities of the Chingaza Park on the first and second axis. Life form overlay shows the tendencies in the vegetation structure on the hydric gradient. 


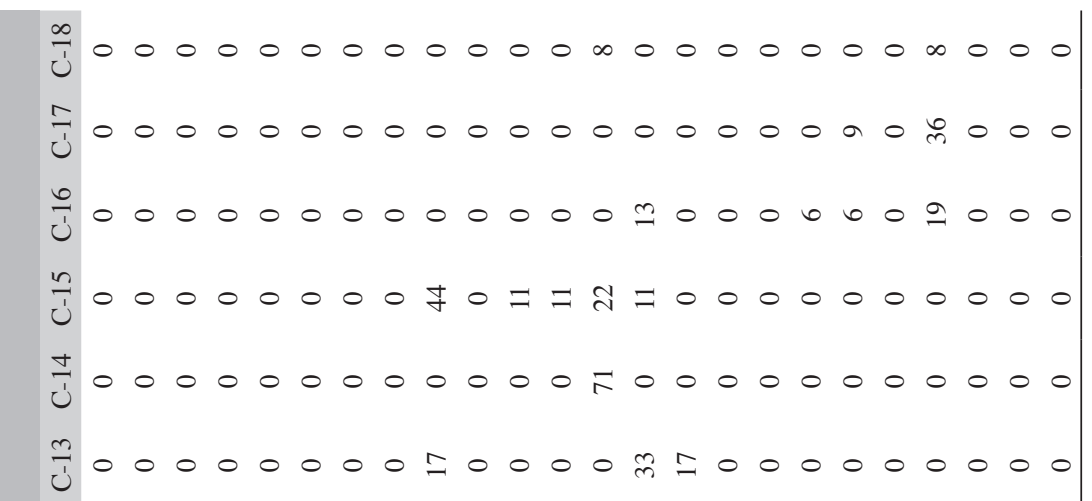

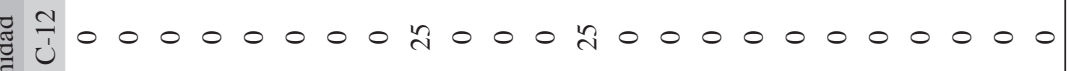
हु

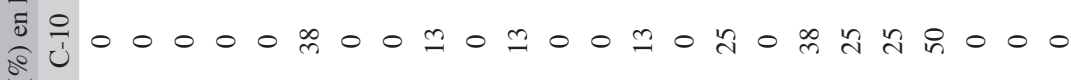
旋

总苗 000000000008000000 in 0 in 000 Uి Uి Uं Uి Uి인

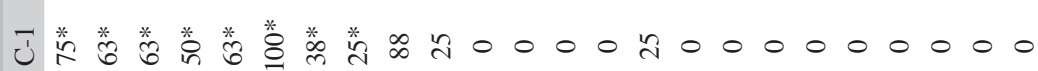




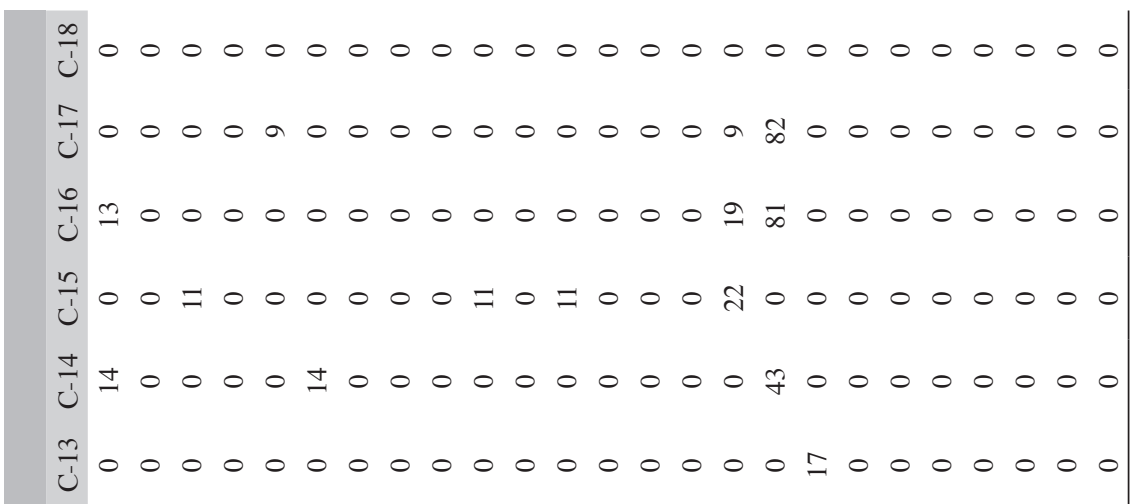
$\frac{1}{1} 0000000000000000000000000$

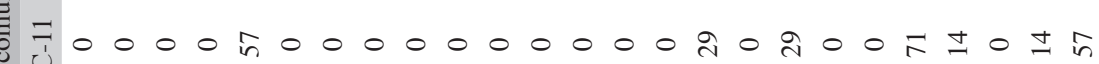

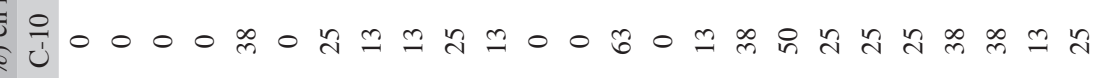
丞

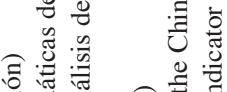
党 苟

嵌

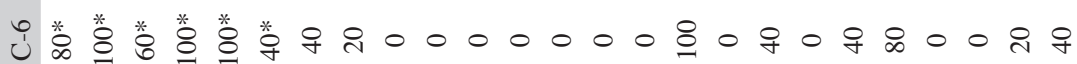
U $00000000000000 m 0 n 0 m 00000$ Uं

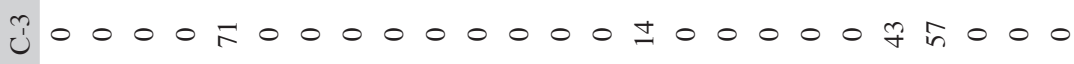
Ũ 000000000000000000000000 Ü口 00 n

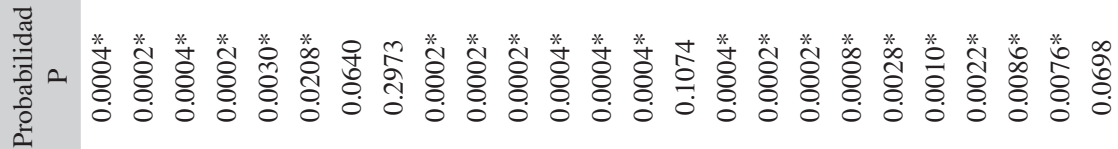




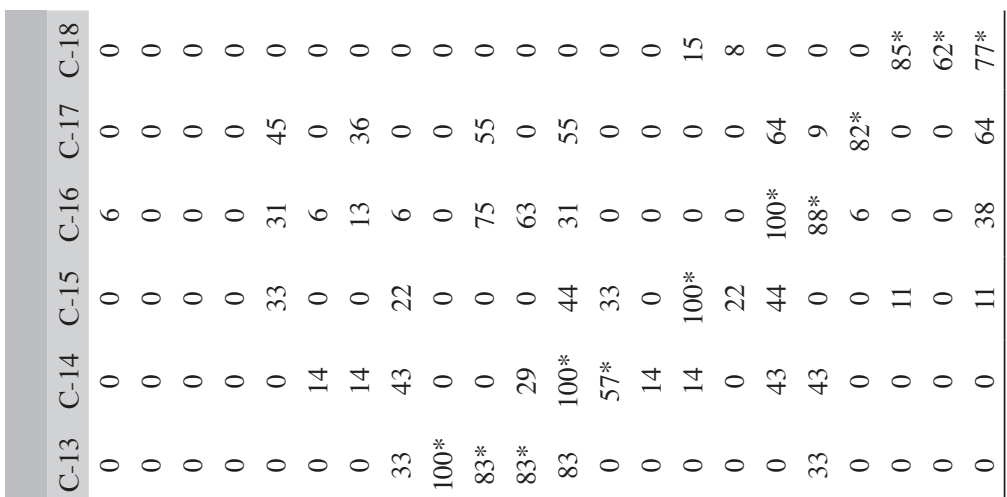

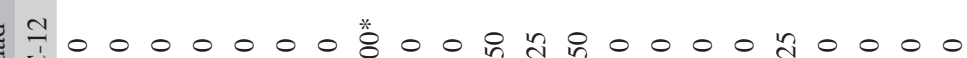
U 苛

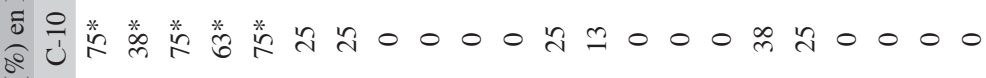
矛 敢 U 幽

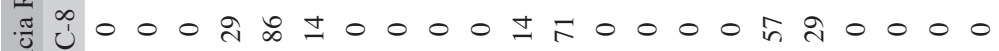

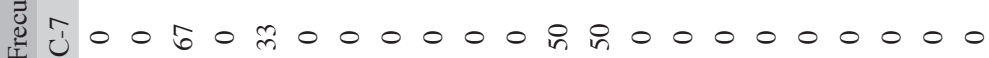
U. un 0 in

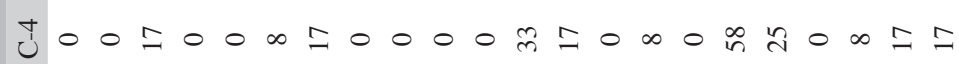
U 000 III 00000000000000000

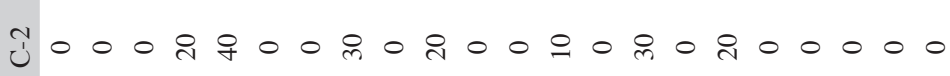
Uं 000 m

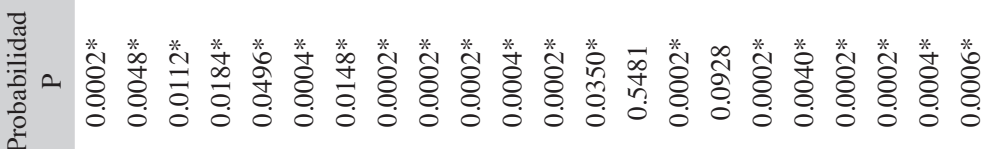
苗 
de las formas helófitos herbáceos y graminoides en un extremo, y helófitos briófitos y juncoides en el otro extremo (Fig. 5). En resumen se puede señalar que la ordenación representa las diferentes zonaciones a lo largo de tres gradientes hídricos principales.

La prueba de Kruskal-Wallis indicó diferencias significativas entre las riquezas medias de las diferentes comunidades $(\mathrm{H}=85.953$; $\mathrm{p}<0.0001$, para un $\alpha=0.05$ ). En general la prueba de comparaciones múltiples de Dunn con corrección de Bonferroni $\left(\alpha^{\prime}=0.0003\right)$ indicó que las comunidades seis, siete y diez presentan una riquezas significativamente mayores a las comunidades dos y 18. Además las riquezas de las comunidades uno, nueve y 11 son mayores a las de la comunidad 18 (Cuadro 2). Esta relación también se puede observar el la ordenación NMDS en donde se presenta una tendencia en la reducción de las riquezas medias desde la vegetación dominada por helófitos graminoides y herbáceos a aquella dominada por plantas sumergidas y juncoides (Fig. 5).
La descripción fisionómica general de la vegetación se basa en las formas de vida dominantes de las especies indicadoras, y las comunidades en la frecuencia relativa de las especies indicadoras principales (Cuadro 1).

C-1. Comunidad de Calamagrostis effusa (Fig. 6 y 10). Representa el $4 \%$ del área muestreada, la riqueza media es de $2.2 \mathrm{spp} /$ grupo. Presente en cuatro transectos de los sectores I y II. Vegetación graminoide de pajonales y chuscales (bambú) dominada por plantas terrestres que toleran un alto contenido de humedad en el suelo. De amplia distribución en el Parque, frecuenta las orillas de turberas, pantanos, charcas y lagunas. Otras especies indicadoras que caracterizan esta comunidad son Aragoa abietina, Chusquea tessellata, Paepalanthus karstenii, Paepalanthus columbiensis, Pernettya prostrata, Breutelia sp. y Geranium sibbaldioides.

C-2. Comunidad de Sphagnum cuspidatum (Fig. 6 y 8). El 10\% del área inventariada corresponde a esta comunidad, la riqueza

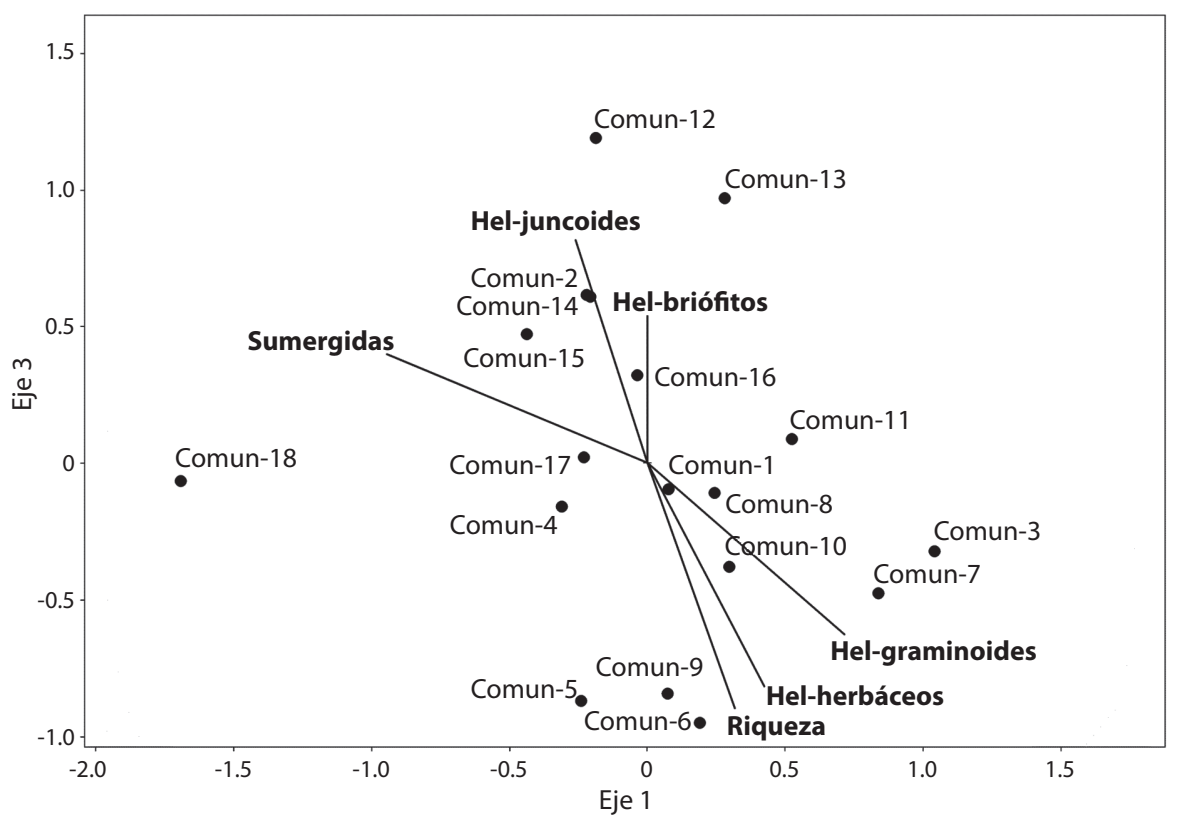

Fig. 5. Ordenación NMDS de las comunidades del PNN Chingaza en el primer y tercer eje. La sobreposición de las formas de vida indica tendencias en la estructura de la vegetación sobre el gradiente hídrico.

Fig. 5. NMDS ordination of communities of the Chingaza Park on the first and third axis. Life form overlay shows the tendencies in the vegetation structure on the hydric gradient. 
CUADRO 2

Prueba Kruskal-Wallis y comparación múltiple de Dunn de la riqueza de especies entre las diferentes comunidades del PNN Chingaza

TABLE 2

Kruskal-Wallis test and Dunn multiple comparisons of species richness between the communities of the Chingaza Park

\begin{tabular}{|c|c|c|c|c|c|c|}
\hline Comunidad & Frecuencia & Suma de los Rangos & Media de los Rangos & & Grupos & \\
\hline Comun-18 & 13 & 265.500 & 20.423 & + & & \\
\hline Comun-12 & 4 & 143.500 & 35.875 & + & + & \\
\hline Comun-2 & 10 & 385.000 & 38.500 & + & + & \\
\hline Comun-3 & 7 & 295.500 & 42.214 & + & + & \\
\hline Comun-14 & 7 & 352.000 & 50.286 & + & + & \\
\hline Comun-15 & 9 & 504.000 & 56.000 & + & + & \\
\hline Comun-13 & 6 & 350.500 & 58.417 & + & + & + \\
\hline Comun-17 & 11 & 741.500 & 67.409 & + & + & + \\
\hline Comun-4 & 12 & 917.500 & 76.458 & + & + & + \\
\hline Comun-16 & 16 & 1225.500 & 76.594 & + & + & + \\
\hline Comun-8 & 7 & 551.000 & 78.714 & + & + & + \\
\hline Comun-11 & 7 & 667.000 & 95.286 & & + & + \\
\hline Comun-1 & 8 & 796.000 & 99.500 & & + & + \\
\hline Comun-5 & 4 & 414.000 & 103.500 & & + & + \\
\hline Comun-9 & 7 & 814.500 & 116.357 & & + & + \\
\hline Comun-10 & 8 & 960.000 & 120.000 & & + & + \\
\hline Comun-7 & 6 & 785.000 & 130.833 & & + & + \\
\hline Comun-6 & 5 & 710.000 & 142.000 & & & + \\
\hline
\end{tabular}

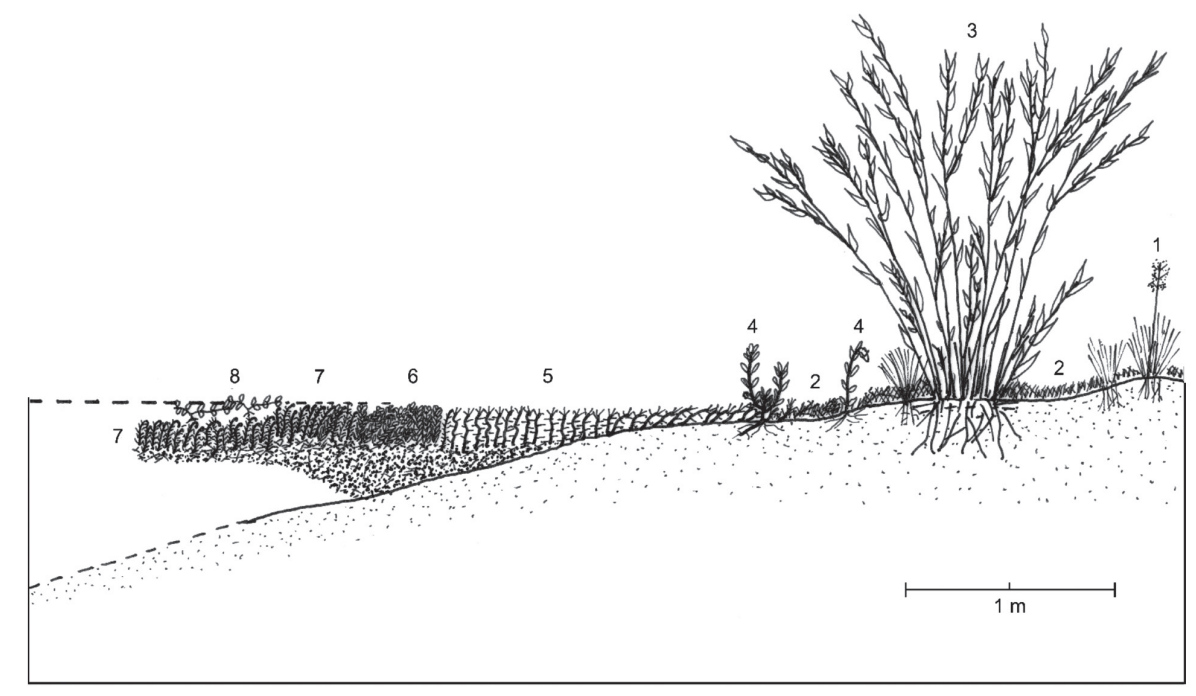

Fig. 6. Perfil de vegetación caracterizado por las comunidades de Calamagrostis effusa (C-1), Sphagnum cuspidatum (C-2) y Elatine paramoana (C-12). 1- Calamagrostis effusa, 2- Pleurozium schreberi, 3- Chusquea tessellata, 4- Bartsia sp., 5Sphagnum cuspidatum, 6- Sphagnum recurvum, 7- Isotachis serrulata, 8- Elatine paramoana.

Fig. 6. Vegetation profile characterized by communities of Calamagrostis effusa (C-1), Sphagnum cuspidatum (C-2), and Elatine paramoana (C-12). 1- Calamagrostis effusa, 2- Pleurozium schreberi, 3- Chusquea tessellata, 4- Bartsia sp., 5Sphagnum cuspidatum, 6- Sphagnum recurvum, 7- Isotachis serrulata, 8- Elatine paramoana. 
media es de 0.9 spp/grupo. Presente en cinco transectos de los sectores I y II. Se presenta de forma sumergida o en colchones emergentes con suelos orgánicos y turbosos al borde de lagunas y charcas. No presenta especies indicadoras adicionales.

C-3. Comunidad de Cyperus rufus. Ocupa el 5\% del área estudiada, la riqueza media es de $1 \mathrm{spp} /$ grupo. Presente en dos transectos del sector II. Vegetación graminoide que caracteriza lugares inundados con suelos minerales $\mathrm{u}$ orgánicos de pantanos y charcas someras. No presenta especies indicadoras adicionales.

C-4. Comunidad de Eleocharis stenocarpa (Fig. 7 y 11). Representa el $7 \%$ del área muestreada, la riqueza media es de $1.6 \mathrm{spp} /$ grupo. Presente en cinco transectos de los sectores I, II y III. La fisionomía se encuentra representada por plantas enraizadas de forma juncoidea típica de pantanos, riberas de cursos de agua corriente, charcas y lagunas. Otra especie indicadora representativa es Polygonum hydropiperoides, la cual también señala condiciones eutróficas.

C-5. Comunidad de Carex acutata. Un $3 \%$ del área estudiada corresponde a esta comunidad, la riqueza media es de $2.1 \mathrm{spp} /$ grupo. Presente en un transecto del sector III. Es caracterizada por plantas graminoides que frecuenta hábitats encharcados con una alto contenido de suelos orgánicos, pantanos y las riberas de lagunas. De acuerdo al grado de inundación se presentan especies indicadoras adicionales como Azolla filiculoides, Carex bonplandii, Ranunculus nubigenus y Phalaris angusta.

C-6. Comunidad de Poa annua. Se presenta en un $3 \%$ del área muestreada, la riqueza media es de 3.9 spp/grupo. Presente en un transecto del sector I. Comunidad ruderal que usualmente se desarrolla sobre suelos alterados. La fisionomía es definida principalmente por plantas graminoides presentes en los pantanos y bordes de lagunas. Especies indicadoras que también caracterizan esta comunidad son Isolepis inundata, Juncus cyperoides, Paspalum cf. vaginatum, Veronica serpyllifolia, Lachemilla sp., Plagiocheilus solivaeformis, Juncus breviculmis y diversos musgos no identificados.

C-7. Comunidad de Valeriana sp. Ocupa el 3\% del área muestreada, la riqueza media es de $3.2 \mathrm{spp} /$ grupo. Presente en un transecto del sector II. Vegetación de suelos húmedos en transición a lugares pantanosos y temporalmente encharcados. Es representada tanto por formas de vida graminoides como herbáceas. Especies indicadoras adicionales son Sisyrinchium bogotense, Halenia asclepiadea, Hypericum juniperinum, Nertera granadensis y Cyperus bipartitus.

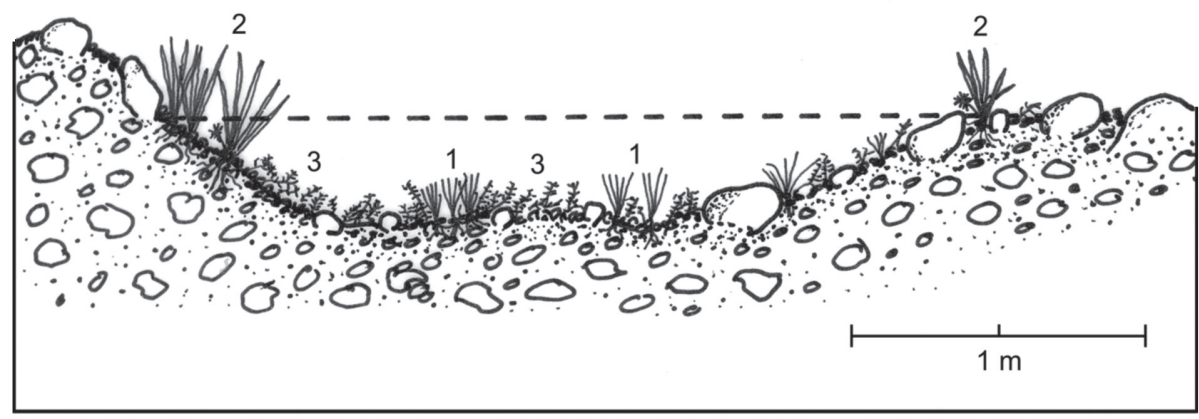

Fig. 7. Perfil de vegetación caracterizado por las comunidades de Eleocharis stenocarpa (C-4) y Crassula venezuelensis. 1- Eleocharis stenocarpa, 2- Juncus breviculmis.

Fig. 7. Vegetation profile characterized by communities of Eleocharis stenocarpa (C-4) and Crassula venezuelensis. 1Eleocharis stenocarpa, 2- Juncus breviculmis. 
C-8. Comunidad de Ranunculus flagelliformis. Representa el 6\% del área muestreada y una riqueza media de $1.6 \mathrm{spp} /$ grupo. Presente en dos transectos del sector II. Esta comunidad ocupa lugares temporales a permanentementes inundados de charcas, bordes de lagunas y bordes de pequeños cursos de agua. Corresponde a una vegetación caracterizada por plantas anfibias de las formas de vida enraizadas herbáceas y en menor grado graminoides. Especie indicadora adicional que también caracteriza esta comunidad es Alopecurus aequalis.

C-9. Comunidad de Carex bonplandii (Fig. 8). Representa el $4 \%$ del área muestreada, la riqueza media es de $2.7 \mathrm{spp} /$ grupo. Presente en cuatro transectos de los sectores I, II y III. La vegetación se encuentra representada por plantas graminoides y herbáceas. Frecuenta los suelos estacionalmente inundados que se presentan a lo largo de los bordes de charcas, lagunas, lagos, quebradas y ríos. Especies indicadoras adicionales que figuran en esta comunidad son Marchantia plicata, Epilobium denticulatum, Lachemilla orbiculata, Hydrocotyle bonplandii, Bidens triplinervia y Paspalum hirtum.

C-10. Comunidad de Festuca andicola. Ocupa el 5\% del área muestreada, la riqueza media es de $3 \mathrm{spp} / \mathrm{grupo}$. Presente en cuatro transectos de los sectores II y III. La fisionomía de la vegetación es caracterizada por plantas de las formas de vida graminoide y herbáceas. Esta comunidad se presenta por lo general sobre un substrato estacional a permanentemente inundado, y caracteriza pantanos con substratos orgánicos, charcas temporal a permanentemente inundados, riberas de pequeños cursos de aguas y los bordes de lagunas. Especies indicadoras adicionales son Anthoxanthum odoratum, Galium ascendens, el musgo Pleurozium schreberi, y Eleocharis macrostachya.

C-11. Comunidad de Muhlenbergia fastigiata. Se presenta en $4 \%$ del área muestreada, la riqueza media es de $1.9 \mathrm{spp} /$ grupo. Presente en tres transectos del sector II. La vegetación se caracteriza por plantas de la forma de vida graminoide y se presenta sobre suelos temporalmente inundados. Frecuenta lugares

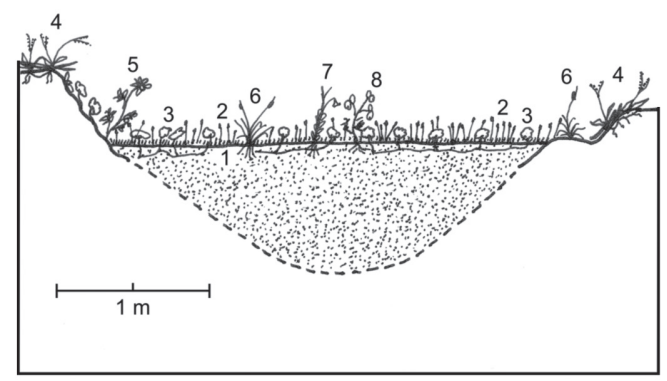

Fig. 8. Perfil de vegetación caracterizado por las comunidades de Carex bonplandii (C-9). 1-Pleurozium schreberi, 2- Eleocharis stenocarpa, 3- Lachemilla orbiculata, 4- Paspalum hirtum, 5- Bidens triplinervia, 6- Carex bonplandii, 7- Epilobium denticulatum, 8Calceolaria mexicana.

Fig. 8. Vegetation profile characterized by communities of Carex bonplandii (C-9). 1- Pleurozium schreberi,

2- Eleocharis stenocarpa, 3- Lachemilla orbiculata, 4- Paspalum hirtum, 5- Bidens triplinervia, 6Carex bonplandii, 7- Epilobium denticulatum, 8Calceolaria mexicana.

pantanosos estacionalmente inundados los cuales se presentan a lo largo de pequeños cursos de agua, charcas y lagunas. Especie indicadora adicional es Carex teres.

C-12. Comunidad de Elatine paramoana (Fig. 6, 9 y 11). Representa el $1 \%$ del área muestreada, la riqueza media es de $0.9 \mathrm{spp} /$ grupo. Presente en cuatro transectos de los sectores I, II y III. Esta comunidad se encuentra representada por plantas herbáceas tanto sumergidas como emergentes, y las cuales crecen en lugares temporal a permanentemente inundados. Por lo general frecuenta charcas de aguas someras estacional a permanentemente inundadas y también lagunas en donde puede llegar a crecer hasta unos $50 \mathrm{~cm}$ de profundidad. No se presenta especie indicadora adicional.

C-13. Comunidad de Isoëtes palmeri (Fig. 10). Ocupa un $4 \%$ del área muestreada, la riqueza media es de $1.3 \mathrm{spp} /$ grupo. Presente en dos transectos de los sectores I y II. Corresponde a una vegetación caracterizada por plantas sumergidas y emergentes de forma juncoide. Se presenta en charcas, pequeñas lagunas y cursos 


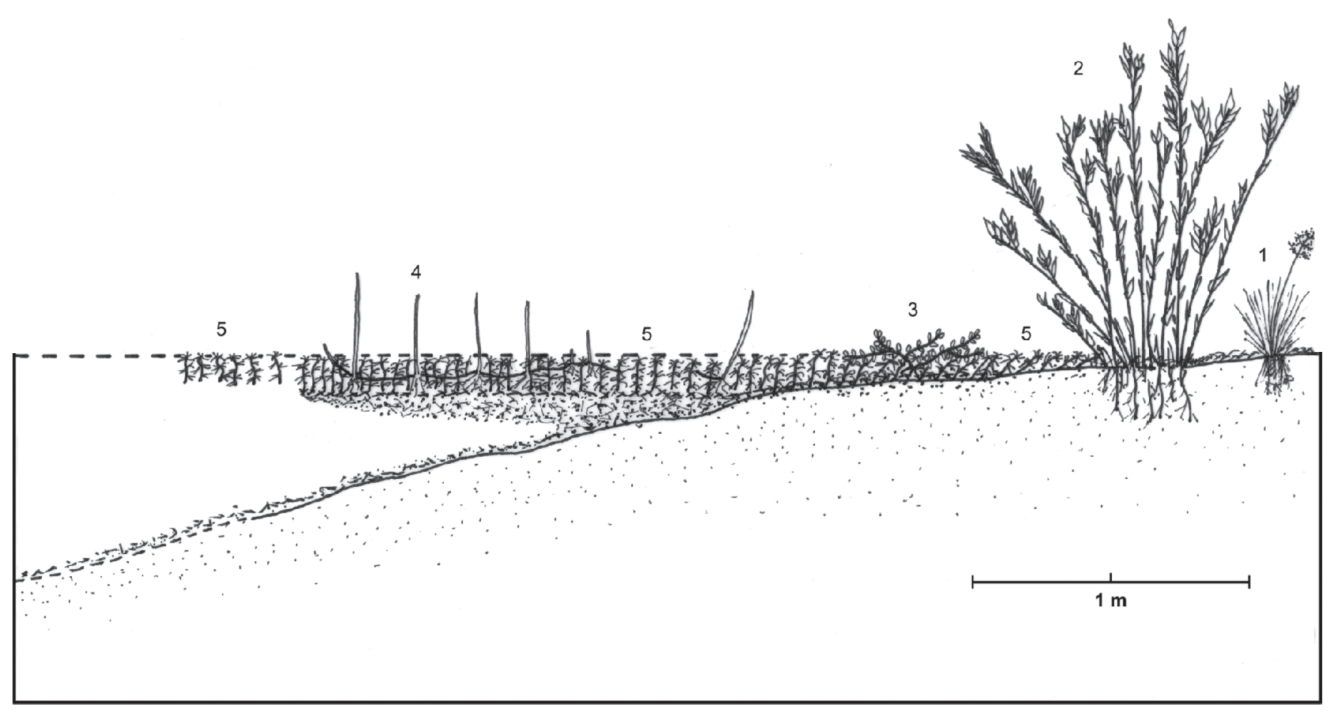

Fig. 9. Perfil de vegetación caracterizado por las comunidades de Sphagnum cuspidatum (C-2) y Elatine paramoana (C-12). 1- Calamagrostis effusa, 2- Chusquea tessellata, 3- Elatine paramoana, 4- Eleocharis macrostachya, 5Sphagnum cuspidatum.

Fig. 9. Vegetation profile characterized by communities of Sphagnum cuspidatum (C-2) and Elatine paramoana (C-12). 1Calamagrostis effusa, 2- Chusquea tessellata, 3- Elatine paramoana, 4- Eleocharis macrostachya, 5- Sphagnum cuspidatum.

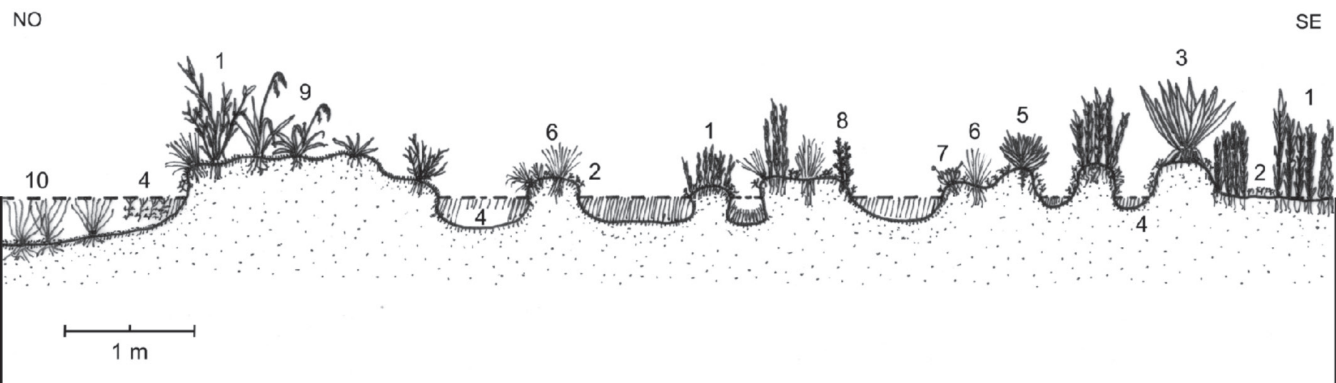

Fig. 10. Perfil de vegetación caracterizado por las comunidades de Calamagrostis effusa (C-1) y Isoëtes palmeri (C-13). 1- Chusquea tessellata, 2- Paepalanthus karstenii, 3- Espeletia grandiflora, 4- Sphagnum cuspidatum, 5- Puya santosii, 6- Calamagrostis effusa, 7- Paepalanthus columbiensis, 8- Aragoa abietina, 9- Carex pichinchensis, 10- Isoëtes palmeri.

Fig. 10. Vegetation profile characterized by communities of Calamagrostis effusa (C-1) and Isoëtes palmeri (C-13). 1- Chusquea tessellata, 2- Paepalanthus karstenii, 3- Espeletia grandiflora, 4- Sphagnum cuspidatum, 5- Puya santosii, 6- Calamagrostis effusa, 7- Paepalanthus columbiensis, 8- Aragoa abietina, 9- Carex pichinchensis, 10- Isoëtes palmeri.

de agua de poca corriente. Especies indicadoras adicionales son Leptodictyum sp. y Juncus ecuadoriensis.

C-14. Comunidad de Crassula venezuelensis (Fig. 7). Se presenta en un 7\% del área muestreada, la riqueza media es de $1.2 \mathrm{spp} /$ grupo. Presente en tres transectos de los sectores I, II y III. Vegetación acuática que se presenta en charcas estacional a permanentemente inundadas, lagunas, lagos y cursos de agua con 
escasa corriente. Se encuentra caracterizada principalmente por plantas sumergidas y herbáceas emergentes. Especie indicadora adicionales es Gratiola bogotensis.

C-15. Comunidad de Lilaeopsis macloviana. El 6\% del área inventariada corresponde a esta comunidad, la riqueza media es de 1.2 spp/grupo. Presente en cinco transectos de los sectores II y III. Vegetación rasante de suelos estacional a permanentemente inundados. Por lo general se encuentra en lugares turbosos con mayor o menor acumulación de materia orgánica. La fisionomía se encuentra definida por plantas emergentes de forma juncoide. No se presenta especie indicadora adicional.

C-16. Comunidad de Callitriche nubige$n a$. Representa el $11 \%$ del área muestreada, la riqueza media es de $1.6 \mathrm{spp} /$ grupo. Presente en cuatro transectos de los sectores II y III. Corresponde a una vegetación acuática representada principalmente por plantas enraizadas de hojas flotantes. Generalmente se presenta en cubetas o charcas de poca profundidad, en la zona litoral de lagunas y lagos, y en cursos de agua con escasa corriente. Especie indicadora adicional es Ranunculus limoselloides.

C-17. Comunidad de Potamogeton paramoanus. Ocupa el 7\% del área muestreada, la riqueza media es de $1.4 \mathrm{spp} /$ grupo. Presente en tres transectos del sector II. La estructura biotipológica de la vegetación se encuentra definida principalmente por plantas enraizadas de hojas flotantes y/o sumergidas. La vegetación representada por plantas de hojas sumergidas y flotantes se encuentra por lo general en lugares con aguas estancadas y de poca profundidad, como son pequeñas charcas o cubetas y la zona litoral de las lagunas. Localmente también en los ríos La Playa-Guatiquia y Chuza, y en riachuelos con escasas a moderadas corrientes de agua en donde se presenta por lo general en su forma sumergida. No se presenta especie indicadora adicional.

C-18. Comunidad de Potamogeton illinoensis (Fig. 11). Se presenta en el $10 \%$ de área muestreada, la riqueza media es de 0.7 spp/grupo. Presente en cuatro transectos del sector III. Esta comunidad se encuentra principalmente en la Laguna de Chingaza, en donde ocupa la zona litoral hasta una profundidad de $4 \mathrm{~m}$. Sin embargo hay que notar que las especies indicadoras adicionales Myriophyllum quitense y Nitella flexilis también se pueden presentar en pequeños riachuelos con escasas a fuertes corrientes de agua.

\section{DISCUSIÓN}

Florística: En un estudio preliminar de la flora del área de estudio Jiménez \& Bernal (1991) registraron 472 especies de fanerógamas para el PNN Chingaza. Sin embargo, en un listado más reciente Rangel (2000b) reconoce 592 especies de plantas vasculares, pero no menciona 26 de las 89 especies de macrófitos acuáticos y semiacuáticos incluidas en el

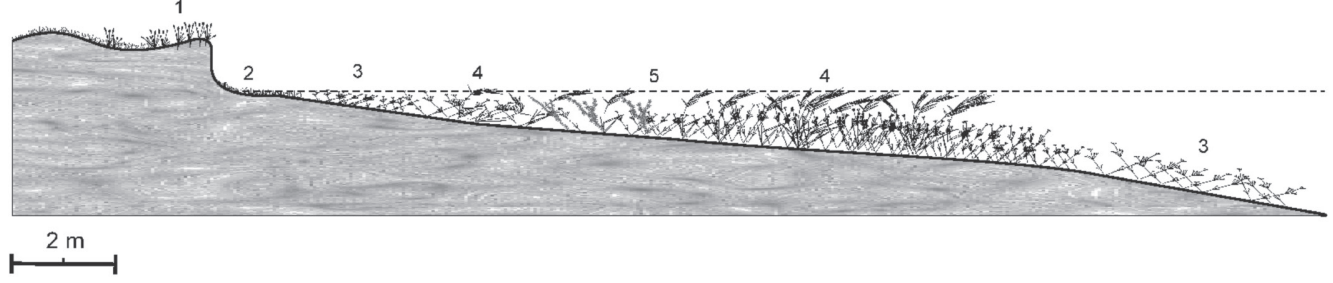

Fig. 11. Perfil de vegetación en la laguna de Chingaza caracterizado por las comunidades de Eleocharis stenocarpa (C-4), Elatine paramoana (C-12) y Potamogeton illinoensis (C-18). 1- Eleocharis stenocarpa, 2- Elatine paramoana, 3- Nitella flexilis, 4- Potamogeton illinoensis, 5- Myriophyllum quitense.

Fig. 11. Vegetation profile characterized by communities of Eleocharis stenocarpa (C-4), Elatine paramoana (C-12), and Potamogeton illinoensis (C-18). 1- Eleocharis stenocarpa, 2- Elatine paramoana, 3- Nitella flexilis, 4- Potamogeton illinoensis, 5- Myriophyllum quitense. 
Apéndice. En un nuevo intento por definir la riqueza florística del Parque, Vargas \& Pedraza (2004) presentan un catálogo actualizado con el registro de 711 especies de plantas vasculares. A este listado se puede agregar cuatro fanerógamas adicionales: Carex acutata, Paspalum cf. vaginatum, Lilaeopsis macloviana y Elatine paramoana. La especie Potamogeton illinoensis, anteriormente registrada por Schmidt-Mumm (1994) para el PNN Chingaza, igualmente no se encuentra incluida en los dos últimos análisis florísticos.

Los briófitos juegan un papel importante en la constitución y procesos de colmatación autóctona de las lagunas someras, charcas o pantanos de las regiones paramunas (Sánchez et al. 1989). Entre los musgos se destacan ampliamente las especies del genero Sphagnum. Siete de las 33 especies registradas por Churchill \& Linares (1995) para Colombia se presentan en el PNN Chingaza. Dada su difícil identificación en el campo las especies sumergidas y de lugares inundados se trataron aquí conjuntamente como Sphagnum cuspidatum/S. recurvum. Otro grupo que presenta frecuentes cambios nomenclaturales corresponde al género Isoëtes, del cual se han registrado en la actualidad cinco especies para el PNN Chingaza (Rangel 2000b, Small \& Hickey 2001).

En cuanto a las angiospermas acuáticas y semiacuáticas en los últimos 15 años se presenta un notorio aumento en la revisión taxonómica de diferentes familias y géneros. Por su relevancia en el reconocimiento de las diferentes comunidades o asociaciones citadas para el Parque, además de sus implicaciones sintaxonómicas, merecen particular aclaración las especies: Elatine paramoana, registradas como E. chilensis Gay. o E. triandra Schkuhr., representa especie nueva para los páramos aledaños a la Sabana de Bogotá (Schmidt-Mumm \& Bernal 1995). Galium ascendens (= G. cuatrecasii Standley) se ha identificada erróneamente como G. canescens Kunth (=G. trianae Wernham) (Dempster 1981, 1982). Material fértil de Lilaeopsis confirman la presencia de L. macloviana para Colombia (Affolter 1985), aunque no se descarta la presencia de $L$. schaffneriana (Schlechtendal) Coulter \& Rose para el páramo de Chingaza. Montia biapiculata se presenta únicamente en los páramos de Sumapaz y de Chingaza (Lourteig 1991).

Comunidades: El análisis de la vegetación de los diversos gradientes terrestreacuáticos del PNN Chingaza resultó en la caracterización de 18 comunidades. Sin embargo, dada la naturaleza del muestreo por transectos, las comunidades relacionadas con el extremo terrestre del gradiente se encuentran evidentemente submuestreadas. Este es el caso de las comunidades de Calamagrostis effusa, Poa annua y Valeriana sp., en donde el óptimo de distribución de las especies indicadoras se encuentra en algún lugar fuera del área muestreada. Por ejemplo, la especie indicadora de la comunidad C-1 (C. effusa) hace parte de diferentes asociaciones de la alianza fitosociológica Paepalantho karsteni-Chusquion tessellatae (Rangel \& Ariza 2000), vegetación que caracteriza los chuscales (páramo de bambú) que se combinan con matorrales y pajonales presente tanto en laderas como en sitios planos con buen contenido de agua en el suelo. En el páramo de Sumapaz se encuentra representada en la alianza Rhynchosporo macrochaeta-Espeletion grandiflorae (Cleef et al. 2008). Así mismo Poa annua es una especie introducida a Colombia como forrajera que actualmente se encuentra distribuida a lo largo de la zona andina en gran parte del país. La posición ecológica de la comunidad de Valeriana sp. (C-7) es incierta mientras no se identifica la especie indicadora.

Una comparación directa de las asociaciones descritas por Franco et al (1986) y Rangel \& Ariza (2000) con aquellas definidas en el presente estudio resulta difícil. Como ya se mencionó en la introducción, la dificultad surge de las diferentes metodologías aplicadas al estudio de la vegetación. El enfoque fitosociológico se centra en el estudio de unidades de vegetación homogéneas, mientras que en el presente estudio el enfoque se encuentra definido por el análisis de los cambios de la vegetación a lo largo de diferentes gradientes hídricos. Implica además tratar con 
una vegetación florística y espacialmente más heterogénea. Con el fin de ilustrar el problema un ejemplo, Franco et al. (1986) describen inicialmente la asociación Caricetum bonplandii para los alrededores de la laguna de Chingaza, asociación que posteriormente fue redefinida por Rangel \& Ariza (2000) como Ranunculo nubigenoris-Caricetum bonplandi. Adicionalmente definen la asociación Hydrocotylo ranunculoidis-Caricetum acutatae (Rangel \& Ariza 2000), con Carex acutata como especie característica exclusiva y Hydrocotyle ranunculoides como característica electiva. En el presente trabajo tanto Ranunculus nubigenus como Carex bonplandii son especies indicadoras adicionales de la comunidad de Carex acutata (C-5), y H. ranunculoides no presentó valor indicador significativo para ninguna de las comunidad aquí descritas. Otro dificultad surge con la asociación Liliaeopsis schaffnerianae-Ranunculetum flagelliformis (Rangel \& Ariza 2000). Como ya se mencionó en la discusión florística, probablemente se trata de Lilaeopsis macloviana en la especie característica electiva de la asociación, la cual además es especie indicadora única de la comunidad de L. macloviana (C-15) descrita en el presente estudio. Igualmente Ranunculus flagelliformis (C-8) representa aquí una comunidad propia. El presente estudio no encuentra evidencia que apoya la inclusión de ambas especies en una sola asociación. Tanto la comunidad de $R$. flagelliformis (C-8) como la de L. macloviana (C-15) presentan marcadas diferencias florísticas (Fig. 3) y de acuerdo a la ordenación (Fig. 4 y 5) ocupan diferentes hábitats sobre el gradiente terrestre-acuático. Problemas similares también se presenta con la asociación Epilobio denticulatae-Cyperetum rufi (Rangel \& Ariza 2000). Aquí las especies indicadoras Cyperus rufus y Epilobium denticulatum hacen parte de las comunidades de $C$. rufus (C-3) y Carex bonplandii (C-9) respectivamente. Por último no sobra mencionar que Rangel \& Ariza (2000) consideran la anterior asociación como parte de la alianza Galio (trianae) canescentis-Gratiolion (peruvianae) bogotensis (Cleef 1981, Cleef et al. 2008), la cual reúne la vegetación de los pantanos y orillas de riachuelos de varios páramos de la cordillera Oriental de Colombia. Sin embargo, como ya se mencionó en las consideraciones florísticas, Galium canescens no es un elemento típico de pantanos, por lo cual dicha alianza debe ser revalidada con G. ascendens como especie diagnóstica de la misma. En cuanto a la vegetación acuática se encontraron concordancias entre las comunidades de Isoëtes palmeri (C-13), Crassula venezuelensis (C-14), Callitriche nubigena (C-16) y Potamogeton illinoensis (C-18) con aquellas descritas por Cleef (1981) y Cleef et al. (2008) para diferentes ambientes acuáticos de los páramos de Colombia. Comunidades que no se han descrito con anterioridad corresponden a Elatine paramoana (C-12), Lilaeopsis macloviana (C-15) y Potamogeton paramoanus (C-17).

Otro de los objetivos del presente trabajo fue el estudio de la zonación de la vegetación a lo largo de diferentes gradientes terrestre-acuáticos. Estos implican datos muy heterogéneos, e independiente del modelo de comportamiento de la vegetación sobre el gradiente, sea este el modelo organísmico de Clements (1936) o el modelo individualista de Gleason (1926), se recomienda primero clasificar y luego ordenar los grupos resultantes para facilitar la interpretación (Matteucci \& Colma 1982). La clasificación de los grupos individuales de cada transecto mediante aglomeración flexible mostró que en un tercio de los transectos los grupos obtenidos con el procedimiento TWINSPAN no presentan una zonación bien definida, los cuales resultaron ser más homogéneos a lo inicialmente esperado. El caso extremo se presentó en la comunidades de Carex acutata (C-5), Poa апnиа (C-6) у Valeriana sp. (C-7), las cuales encuentran representación a lo largo de un solo transecto. Por otro lado un $47 \%$ de los transectos mostraron una zonación definida apenas por dos comunidades y el restante $20 \%$ por tres a cuatro comunidades. Estudios más recientes, en los cuales se analizó la distribución de las especies a lo largo de diferentes gradientes hídricos, han mostrado que los modelos organísmicos e individualistas resultan ser simplistas ya que no lograron explicar los diferentes patrones 
de zonación encontrados (Keddy 1983, 1984, 2000). Shipley \& Keddy (1987) y Hoagland \& Collins (1997), nuevamente confirman el anterior punto de vista y sugieren que se requieren más de un modelo para explicar la estructura de la vegetación a lo largo de gradientes hídricos. Igualmente proponen la necesidad de explorar al menos cuatro modelos alternativos que puedan explicar los patrones de distribución de las especies. Dado el tamaño del área de los cuadrados utilizados en los transectos del presente estudio no es posible definir modelos del patrón de zonación de la vegetación acuática y semiacuática en el PNN Chingaza. Sin embargo, la presencia de transectos que no exhiben zonación alguna o de aquellos que muestran de dos a cuatro franjas de zonación sugiere la existencia de diferentes patrones de distribución de las especies a lo largo de los gradientes hídricos estudiados en el parque.

Particularmente en los trópicos la descripción, clasificación y el mapeo de la vegetación continúa siendo una actividad importante de la ecología vegetal. Una comprensión adecuada de la vegetación sirve como base para el adecuado uso y manejo del paisaje natural, para la estimación de la capacidad productora de zonas o regiones particulares, y para la predicción de las consecuencias resultantes de determinadas actividades humanas. En años recientes esta última también incluye los cambios generados por el calentamiento global. Uno de los objetivos del actual trabajo es el de retomar una idea anteriormente expresada por Whittaker (1970), quien afirma que la clasificación y el análisis de gradientes son actividades complementarias y además se pueden combinar para aumentar la efectividad de ambos.

En cuanto a gradientes de profundidad, se puede observar que la mayoría de los transectos se realizaron en sistemas lagunares o humedales que no presentaron vegetación sumergida a profundidades mayores de los $50 \mathrm{~cm}$. Una excepción se encontró en la laguna de Chingaza, la cual se caracteriza por sus aguas oligotróficas, ácido-blandas, transparencias Secchi entre 3.5 y $6.5 \mathrm{~m}$ y profundidades medias de $17 \mathrm{~m}$ (máxima $27 \mathrm{~m}$ en invierno)
(Gaviria 1993; Donato et al. 1996). Aquí las mayores profundidades colonizadas por macrófitos, principalmente Nitella flexilis, fue de unos 4m (Fig. 11). En comparación el Lago de Tota, localizado a una altura de 3 050m.s.n.m., registra profundidades de hasta $22 \mathrm{~m}$ para $N$. flexilis y $12 \mathrm{~m}$ para $N$. clavata Kützing, Myriophyllum quitense y Potamogeton illinoensis (Rangel \& Aguirre 1983). En general Cleef (1981) menciona, sin especificar el lugar, una profundidad aproximada de $3 \mathrm{~m}$ para la alianza Potameto-Myriophyllion (elatinoides) quitensis en lagunas del subpáramo. En la represa del Neusa, localizada a 2 952m.s.n.m., Carrillo et al. (2006) registran profundidades máximas de $7 \mathrm{~m}$ para Egeria densa Planchon. Kiersch et al. (2004) observaron en Ecuador la presencia de $M$. quitense a $2 \mathrm{~m}$ y $P$. illinoensis a $3.5 \mathrm{~m}$ de profundad en la laguna de La Mica, localizada a 3 900m.s.n.m. En la misma laguna Stuckenia (Potamogeton) striata (Ruiz \& Pav.) Holub coloniza profundidades de $4.3 \mathrm{~m}$ y Elodea (matthewsii) potamogeton (Bertero) Espinosa de $6 \mathrm{~m}$. Para el Lago Cuicocha, a 3 064m.s.n.m, los mismos autores registran la presencia de $M$. quitense a $2.3 \mathrm{~m}$ y P. illinoensis a $8 \mathrm{~m}$ de profundidad. Diversas especies de Chara y Nitella llegan inclusive a profundidades de 30 a $35 \mathrm{~m}$. Se desconoce la razón de las pocas profundidades colonizadas por los macrófitos en la Laguna de Chingaza. Las condiciones oligotróficas de la laguna no parecen ser el factor limitante, aunque se requieren de estudios detallados de las condiciones físicas y químicas del sedimento para definir el substrato como posible elemento limitante. Spencer (1982) considera la luz como factor determinante que limita la distribución de profundidad de las plantas acuáticas. A pesar de la buena penetración luminosa en la laguna, con profundidades disco Secchi entre 3.5 y $6.5 \mathrm{~m}$ (Gaviria 1993), es conveniente considerar la frecuente y alta nubosidad que se presenta en el páramo de Chingaza a lo largo del año como elemento limitante adicional. En el PNN Chingaza la insolación varia de 2.5 a $3.5 \mathrm{~h}$ de brillo solar al día, con los meses más radiantes situados entre noviembre y febrero y los más nublados entre junio y julio (Vargas \& 
Pedraza 2004). Tanto el Lago de Tota como la represa del Neusa se encuentran localizados en zonas climáticas más secas, por lo cual reciben una mayor radiación solar por día en el año.

Contrario a la laguna de Chingaza, el embalse de Chusa no presenta una vegetación acuática o semiacuática bien desarrollada. En gran parte del embalse la zona litoral presenta fuertes pendientes la cual además se encuentra sometida a marcados cambios en el nivel del agua. Los cambios del nivel del agua son irregulares, dependen de la demanda de agua y de las precipitaciones, y de acuerdo a Donato et al. (1996) pueden llegar a ser del orden de los $25 \mathrm{~m}$ entre los meses de mayo a septiembre. Este evento destructivo ha generado en el embalse de Chuza una zona litoral desprovista de vegetación y susceptible a los efectos de la erosión.

Por otro lado la vegetación de pantano y de las zonas litorales de lagos y lagunas del PNN Chingaza frecuentemente presenta signos de disturbios. En gran parte estos disturbios son generados por el pastoreo y pisoteo de ganado vacuno y recientemente también por la recuperación de las poblaciones del venado cola blanca (Odocoileus virginianus), y en menor grado por el uso de fuego para la mejora en la calidad del forraje. Las áreas húmedas y pantanosas fuertemente influenciadas por la presencia de ganado tienden a presentar un aumento de musgos (Breutelia sp.) y ciperáceas (Vargas et al. 2002). Especies indicadoras de este régimen de disturbios son Lachemilla orbiculata, Paspalum hirtum, Trifolium repens, Carex bonplandii y Eleocharis acicularis (Vargas \& Rivera 1991, Cárdenas et al. 2002, Premauer \& Vargas 2004). De acuerdo a lo anterior y observaciones de campo se puede afirmar que las comunidades de Carex acutata (C-5), Poa апnиа (C-6), Carex bonplandii (C-9) у Festuca andicola (C-10) son en gran parte el producto de alteraciones del medio generados por la recurrente presencia de ganado.

Un caso particular de herbivoría se observó en la comunidad de Lilaeopsis macloviana (C-15). En todas las poblaciones de L. macloviana que crecían sobre substratos turbosos, algunas de ellas con extensiones de hasta unos
$100 \mathrm{~m}^{2}$, las plantas presentaban colchones compactos de escasos centímetros de altura con apariencia de céspedes podados. A pesar de la presencia de ganado vacuno en las aéreas estudiadas no se logro evidenciar alteraciones del suelo por pisoteo, lo cual excluye un posible pastoreo por el mismo. Aunque, no se logró observar directamente la presencia del curí o cuy (Cavia porcellus), esta especie resulta ser de hábito nocturno y bastante esquiva, la gran cantidad de caminos encontrados a lo largo de las comunidades de L. macloviana sugieren que estos sirven como lugares importantes de pastoreo. Sin embargo, se requieren experimentos de exclusión de los herbívoros para determinar el grado de impacto que el curí tiene sobre el crecimiento vertical de las comunidades de L. macloviana. En áreas pantanosas del páramo $C$. porcellus también forrajea las hojas de Carex jamesonii Boott y otras ciperáceas (Cleef 1981).

Otro tipo de evento destructivo se generó en el valle del Río la Playa durante la extracción de material de construcción para carreteras y la Represa de Chuza, la cual finalizó en el año 1983. Durante esta fase de construcción se generaron una serie de excavaciones que posteriormente se llenaron de agua para dar lugar a pequeñas cubetas y charcas de poca profundidad. Estos cuerpos de agua presentan un substrato mineral usualmente formado por material pedregoso a arenoso. Algunos de ellos pueden mantener un espejo de agua durante todo el año, otros se secan parcial o totalmente durante la época seca. Los macrófitos acuáticos y semiacuáticos que lograron colonizar estos nuevos hábitats corresponden en general a las comunidades de Eleocharis stenocarpa (C-4), Ranunculus flagelliformis (C-8), Elatine paramoana (C-12), Isoëtes palmeri (C-13), Crassula venezuelensis (C-14) y Callitriche nubigena (C-16).

Frecuentemente, se ha documentado el alto grado de deterioro generado por la agricultura, las quemas y la ganadería en que se encuentran los páramos de Colombia (Vargas 1996, 1997; Rangel 2000a). El páramo de Chingaza no representa una excepción a este fenómeno y 
resulta importante mencionar que prácticamente todos los hábitats acuáticos y de pantanos estudiados, con excepción de aquellos localizados en la zona limnética de lagos y lagunas, presentan diferentes grados de alteración generada por las actividades humanas directas o indirectas. Particular atención merecen aquí los depósitos turbosos formados por diferentes especies de Sphagnum (comunidades de $S$. cuspidatum entre otras), los cuales representan verdaderos reservorios de agua importantes para el mantenimiento del recurso hídrico del PNN Chingaza (Torres 1985). Las turberas son consideradas como sumideros importantes del carbono atmosférico (Moore 2002) y además ecosistemas estables a largo plazo (Gunnarsson et al. 2002). Las especies del género Sphagnum son consideradas ingenieros ecológicos al secuestrar nutrientes como el nitrógeno y el fósforo de la rizosfera y crear ambientes hostiles para la mayoría de las plantas vasculares (van Breemen 1995, Wiedermann et al. 2009). Igualmente producen una necromasa resistente a la descomposición por lo cual incrementan la tasa de acumulación de turba (Malmer et al. 2003). Sin embargo, estudios recientes realizados en Europa (Malmer \& Wallén 2004, Franzén 2006) y en Norteamérica (Bubier et al. 2007) sugieren que las turberas boreales, enriquecidas con entradas de nitrógeno a través de escorrentía y precipitación atmosférica, están cambiando de su estado inmovilizador de carbono a un estado liberador de carbono. Por otro lado, si la entrada de nitrógeno y fósforo sobrepasa la capacidad de acumulación de las especies de Sphagnum, estos nutrientes son lixiviados y se encuentran disponibles para las plantas vasculares, las cuales a su vez aumentan su productividad y disminuyen así el crecimiento de Sphagnum. De esta manera la polución con nitrógeno tiende a reducir la tasa de acumulación de necromasa y la capacidad de las turberas para retener el carbono (Malmer et al. 2003). Problemas similares aparentemente no han afectado aún las turberas de la Patagonia chilena (Kleinebecker et al. 2008). Sin embargo, los porcentajes relativamente altos de nitrógeno registrados por Sánchez et al. (1989) para algunos páramos circundantes a la Sabana de Bogotá, como también los aumentos periódicos de las concentraciones de fósforo en la laguna de Chingaza probablemente relacionadas con las quemas del páramo (Donato 1991, Donato et al. 1996), hacen temer un proceso de deterioro de los ecosistemas dominados por Sphagnum en el PNN Chingaza. Los anteriores procesos no se han estudiado para las turberas de los páramos colombianos y el constante aumento en la utilización de los recursos hídricos del páramo de Chingaza para consumo humano requiere la pronta investigación de estos fenómenos con el fin de manejar dichos recursos de manera sostenible.

\section{AGRADECIMIENTOS}

A Germán Quintiaquez por la ayuda prestada durante la fase de campo, Edgar Linares por su colaboración en la identificación de los musgos. Contribución del "Programa de Investigaciones para el Desarrollo de la Ecología y la Sistemática en la Pontificia Universidad Javeriana" (Proyecto Colciencias No. 1203-05-013-87). A los revisores anónimos por sus sugerencias y comentarios realizados al manuscrito.

\section{RESUMEN}

La vegetación acuática y semiacuática de los páramos andinos ha sido estudiada generalmente bajo un enfoque fitosociológico tradicional, el cual se basa en muestreos de áreas homogéneas y excluye los fenómenos de borde o transicionales. En el presente estudio se analizó la vegetación acuática y semiacuática del Parque Nacional Natural Chingaza a lo largo de diferentes gradientes hídricos. Asimismo se registran un total de 89 especies en 30 transectos; mediante clasificación numérica y el análisis de especies indicadoras se caracterizan las siguientes 18 comunidades: 1) Calamagrostis effusa, 2) Sphagnum cuspidatum, 3) Cyperus rufus, 4) Eleocharis stenocarpa, 5) Carex acutata, 6) Poa annua, 7) Valeriana sp., 8) Ranunculus flagelliformis, 9) Carex bonplandii, 10) Festuca andicola, 11) Muhlenbergia fastigiata, 12) Elatine paramoana, 13) Isoëtes palmeri, 14) Crassula venezuelensis, 15) Lilaeopsis macloviana, 16) Callitriche nubigena, 17) Potamogeton paramoanus y 18) Potamogeton illinoensis. La ordenación de las comunidades indica la presencia de tres gradientes terrestre-acuáticos diferentes, los cuales se relacionan con 
las formas de vida de las especies que caracterizan las comunidades. Además se considera que gran parte de la heterogeneidad presentada por la vegetación es el resultado de las alteraciones ambientales generadas por diversas actividades humanas (quemas, ganadería, extracción de material para la construcción de carreteras y la represa).

Palabras clave: Andes, comunidades acuáticas, gradiente terrestre-acuático, macrófitos, pantanos, páramo, zonación.

\section{REFERENCIAS}

Addinsoft. 2008. XLSTAT-Pro Version 2008. Statistical Software for MS Excel. http://www.xlstat.com/.

Affolter, J.M. 1985. A monograph of the genus Lilaeopsis (Umbelliferae). Syst. Bot. Monogr. 6: 1-140.

Braun-Blanquet, J. 1979. Fitosociología, bases para el estudio de las comunidades vegetales. Blume, Madrid, España.

Bubier, J.L., T.R. Moore \& L.A. Bledzki. 2007. Effects of nutrient addition on vegetation and carbon cycling in an ombrotrophic bog. Global Change Biol. 13: 1168-1186.

Cárdenas, C., C. Posada \& O. Vargas. 2002. Banco de semillas germinable de una comunidad vegetal de páramo húmedo sometida a quema y pastoreo (Parque Nacional Natural Chingaza, Colombia). Ecotrópicos 15: 51-60.

Cárdenas, C. \& O. Vargas. 2008. Rasgos de historia de vida de especies en una comunidad vegetal alterada en un páramo húmedo (Parque Nacional Natural Chingaza). Caldasia 30: 245-264.

Carrillo, Y., A. Guarín \& G. Guillot. 2006. Biomass distribution, growth and decay of Egeria densa in a tropical high-mountain reservoir (Neusa, Colombia). Aquat. Bot. 85: 7-15.

Carter, V., P.T. Gammon \& M.K. Garrett. 1994. Ecotone dynamics and boundary determination in the Great Dismal Swamp. Ecol. Appl. 4: 189-303.

Churchill, P.S. \& E.L. Linares. 1995. Prodromus Bryologiae Novo-Granatensis. Introducción a la flora de musgos de Colombia. Parte 1: Adelotheciaceae a Funariaceae, Bibl. J.J. Triana 12: 1-453. Parte 2: Grimmiaceae a Trachypodiaceae, Bibl. J.J. Triana 12: 455-924.Instituto de Ciencias Naturales - Museo de Historia Natural, Facultad de Ciencias, Universidad Nacional de Colombia, Bogotá, Colombia.

Cleef, A.M. 1981. The vegetation of the páramos of the Colombian Cordillera Oriental. Dissertationes
Botanicae, Band 61. Cramer, Vaduz, Principado de Liechtenstein.

Cleef, A.M. 2008. Humid cloud superparamo probably acts as a plant diversity centre and as a cool refuge: the case of Nevado de Sumapaz, Colombia, p. 565-593. In T. van der Hammen (ed.). La Cordillera Oriental Colombiana, transecto Sumapaz. Studies on Tropical Andean Ecosystems 7. Cramer, Berlín, Alemania.

Cleef, A.M., J.O. Rangel-Ch. \& H. Arellano. 2008. The páramo vegetation of the Sumapaz massif (Eastern Cordillera, Colombia), p. 799-913. In T. van der Hammen (ed.). La Cordillera Oriental Colombiana, transecto Sumapaz. Studies on Tropical Andean Ecosystems 7. Cramer, Berlín, Alemania.

Clements, F.E. 1936. Nature and structure of climax. J. Ecol. 24: 254-282.

Cuello, N.L. \& A.M. Cleef. 2009. The páramo vegetation of Ramal de Guaramacal, Trujillo State, Venezuela. 2. Azonal vegetation. Phytocoenologia 39: 389-409.

Danserau, P. 1959. Vascular aquatic plant communities of southern Quebec: a preliminary analysis. Transactions of the Northeast Wildlife Conference 10: 27-54.

Davis, M.M., S.W. Sprecher, J.S. Wakeley \& G.R. Best. 1996. Environmental gradients and identification of wetlands in North-central Florida. Wetlands 16: 512-523.

Dempster, L.T. 1981. The genus Galium (Rubiaceae) in South America, II. Allertonia 2: 393-426.

Dempster, L.T. 1982. The genus Galium (Rubiaceae) in South America, III. Allertonia 3: 211-258.

Donato, J. 1991. Fitoplancton y aspectos físicos y químicos de la laguna de Chingaza en Cundinamarca, Colombia. Caldasia 16: 489-500.

Donato, J. 2001. Fitoplancton de los lagos andinos del norte de Sudamérica (Colombia): Composición y factores de distribución. Colección Jorge Álvarez Lleras No. 19. Academia Colombiana de Ciencias Exactas, Físicas y Naturales, Bogotá, Colombia.

Donato, J., L.E. Gonzalez \& C.L. Rodriguez. 1996. Ecología de dos sistemas acuáticos de páramo. Colección Jorge Álvarez Lleras No. 9. Academia Colombiana de Ciencias Exactas, Físicas y Naturales, Bogotá, Colombia.

Dufrêne, M. \& P. Legendre. 1997. Species assemblages and indicator species: the need for a flexible asymmetrical approach. Ecol. Monogr. 67: 345-366. 
Franco, P., O. Rangel-Ch. \& G. Lozano. 1986. Estudios ecológicos en la Cordillera Oriental, II: Las comunidades vegetales de los alrededores de la Laguna de Chingaza (Cundinamarca). Caldasia 15: 219-248.

Franzén, L.G. 2006. Increased decomposition of subsurface peat in Swedish raised bogs: are temperate peatlands still net sinks of carbon? Mires and Peat 1: 1-16 (Also available on line: http://www.mires-and-peat. net/map01/map_1_3.pdf).

Gaviria, S. 1993. Aspectos limnológicos de las lagunas de Chingaza, p. 189-205. In G.I. Andrade (ed.) Carpanta, selva nublada y páramo. Fundación Natura Colombia, Bogotá, Colombia.

Gleason, H.A. 1926. The individualistic concept of the plant association. Bull. Torrey Bot. Club 53: 7-26.

Gunnarsson, U., N. Malmer \& H. Rydin. 2002. Dynamics or constancy in Sphagnum dominated mire ecosystems? A 40-year study. Ecography 25:685-704.

Hill, M.O. 1979. TWINSPAN - a FORTRAN program for arranging multivariate data in an ordered two way table by classification of the individuals and the attributes. Cornell University, Department of Ecology and Systematics, Ithaca, Nueva York, EEUU.

Hill, M.O., R.G.H. Bunce \& M.W. Shaw. 1975. Indicator species analysis, a divisive polythetic method of classification and its application to a survey of native pinewoods in Scotland. J. Ecol. 63: 597-613.

Hoagland, B.W. \& S.L. Collins. 1997. Gradient models, gradient analysis, and the hierarchical structure in plant communities. Oikos 78: 23-30.

Hofstede, R. 1995. Effects of burning and grazing on a colombian paramo ecosystem. Ph.D. Thesis, University of Amsterdam, Ámsterdam, Holanda.

Hofstede, R., M.X. Mondragón \& C.M. Rocha. 1995. Biomass of grazed, burned, and undisturbed páramo grasslands, Colombia. I. Above ground vegetation. Artic Alp. Res. 27: 1-12.

Holland, M.M. 1996. Wetlands and environmental gradients, p. 19-43. In G. Mulamoottil, B.G. Warner \& E.A. Mcbean (eds.). Wetlands: environmental gradients, boundaries, and buffers. CRC, Boca Raton, Florida, EEUU.

Hutchinson, G.E. 1975. A treatise on limnology, III: Limnological botany. Wiley, Nueva York, EEUU.

Jensén, S. \& E. van der Maarel. 1980. Numerical approaches to lake classification with special reference to macrophyte communities. Vegetatio 42: 117-128.
Jiménez, L.C. \& H.Y. Bernal. 1991. Flora fanerogámica del Parque Nacional Natural Chingaza, I: Resultados preliminares. Cuadernos Divulgativos Univ. Javeriana 15: 1-30.

Kautsky, H. \& E. van der Maarel. 1990. Multivariate approaches to the variation in phytobenthic communities and environmental vectors in the Baltic Sea. Mar. Ecol. Prog. Ser. 60: 169-184.

Keddy, P.A. 1983. Shoreline vegetation in Axe Lake, Ontario: effects of exposure on zonation patterns. Ecology 64: $331-344$

Keddy, P.A. 1984. Plant zonation on lakeshores in Nova Scotia: a test of the resource specialization hypothesis. J. Ecol. 72: 797-808.

Keddy, P.A. 2000. Wetland ecology, principles and conservation. Cambridge University, Cambridge, Inglaterra.

Kiersch, B., R. Mühleck \& G. Gunkel. 2004. Las macrófitas de algunos lagos alto-andinos del Ecuador y su bajo potencial como bioindicadores de eutrofización. Rev. Biol. Trop. 52: 829-837.

Kirkman, K., M.B. Drew, L.T. West \& E.R. Blood. 1998. Ecotone characterization between upland longleaf Pine/Wiregrass stands and seasonally-ponded isolated wetlands. Wetlands 18: 346-364.

Kleinebecker, T., N. Hölzel \& A. Vogel. 2008. South Patagonian ombrotrophic bog vegetation reflects biogeochemical gradients at the landscape level. J. Veg. Sci. 19: 151-160.

Legendre, P. \& L. Legendre. 1998. Numerical ecology. Elsevier Science B.V., Ámsterdam, Holanda.

Lourteig, A. 1991. El género Montia (Portulacaceae) en el Hemisferio Austral. Rev. Acad. Colomb. Cien. 18: 41-48.

Luken, J.O. \& T.N. Bezold. 2000. Plant communities associated with different shoreline elements at Cave Run Lake, Kentucky. Wetlands 20: 479-486.

Luteyn, J.L. 1999. Páramos a checklist of plant diversity, geographical distribution, and botanical literature. Mem. New York Bot. Gard. 84: 1-278.

Malmer, N. \& B. Wallén. 2004. Input rates, decay losses and accumulation rates of carbon in bogs during the last millennium: internal processes and environmental change. The Holocene 14: 111-117.

Malmer, N., C. Albinsson, B.M. Svensson \& B. Wallén. 2003. Interferences between Sphagnum and vascular 
plants: effects on plant community structure and peat formation. Oikos 100: 469-482.

Matteucci, S.D. \& A. Colma. 1982. Metodología para el estudio de la vegetación. Secretaría General de la Organización de los Estados Americanos, Washington, D.C., EEUU.

McCune, B. \& J.B. Grace. 2002. Analysis of ecological communities. MjM Software Design, Gleneden Beach, Oregon, EEUU.

McCune, B. \& M.J. Mefford. 2006. PC-ORD. Multivariate Analysis of Ecological Data. Version 5.18. MjM Software Design, Gleneden Beach, Oregon, EEUU.

Molinillo, M. \& M. Monasterio. 2002. Patrones de vegetación y pastoreo en ambientes de páramo. Ecotrópicos 15: 19-34.

Moore, P.D. 2002. The future of cool temperate bogs. Environ. Conservat. 29: 3-20.

Premauer, J. \& O. Vargas. 2004. Patrones de diversidad en vegetación pastoreada y quemada en un páramo húmedo (Parque Natural Chingaza, Colombia). Ecotrópicos 17: 52-66.

Rangel-Ch., J.O. 2000a. La región paramuna y franja aledaña en Colombia, p. 1-23. In J.O. Rangel Ch. (ed.) Colombia, diversidad biótica III: La región de vida paramuna. Universidad Nacional de Colombia, Bogotá, Colombia.

Rangel-Ch., J.O. 2000b. Catálogo florística de los macizos de Chingaza y Sumapaz, p. 563-598. In J.O. RangelCh. (ed.). Colombia diversidad biótica III: La región de vida paramuna. Universidad Nacional de Colombia, Bogotá, Colombia.

Rangel-Ch., J.O. 2000c. La diversidad beta: tipos de vegetación, p. 658-719. In J.O. Rangel-Ch. (ed.). Colombia diversidad biótica III: La región de vida paramuna. Universidad Nacional de Colombia, Bogotá, Colombia.

Rangel-Ch., J.O. 2000d. Síntesis final: visión integradora sobre la región del páramo, p. 814-836. In J.O. Rangel-Ch. (ed.). Colombia diversidad biótica III: La región de vida paramuna. Universidad Nacional de Colombia, Bogotá, Colombia.

Rangel-Ch., J.O. \& J. Aguirre. 1983. Comunidades acuáticas alto-andinas I: Vegetación sumergida y de ribera en el Lago de Tota, Boyacá, Colombia. Caldasia 13: 719-742.
Rangel-Ch., J.O. \& C. Ariza-N. 2000. La vegetación del Parque Nacional Natural Chingaza, p. 720-753. In J.O. Rangel-Ch. (ed.). Colombia diversidad biótica III: La región de vida paramuna. Universidad Nacional de Colombia, Bogotá, Colombia.

Sánchez-M., R., J.O. Rangel-Ch. \& J. Aguirre. 1989. Estudios ecológicos en la Cordillera Oriental, IV: Aspectos sinecológicos de la Brioflora de los depósitos turbosos paramunos de los alrededores de Bogotá. Caldasia 16: 41-57.

Sánchez-M., R. \& J.O. Rangel-Ch. 1990. Estudios ecológicos en la Cordillera Oriental colombiana, V: Análisis fitosociológico de la vegetación de los depósitos turbosos paramunos de los alrededores de Bogotá. Caldasia 16: 155-191.

Schmidt-Mumm, U. 1994. Potamogetonaceae en la flora vascular acuática del Parque Nacional Natural Chingaza, Colombia. Universitas Scientiarium 2: 45-56.

Schmidt-Mumm, U. \& H.Y. Bernal. 1995. A new species of Elatine (Elatinaceae) from the Colombian paramos in the northern Andes. Brittonia 47: 27-30.

Sculthorpe, C.D. 1967. The biology of aquatic vascular plants. Koeltz, Königstein, Alemania.

Shipley, B. \& P.A. Keddy. 1987. The individualistic and community-unit concepts as falsifiable hypotheses. Vegetatio 69: 47-55.

Small, R.L. \& R.J. Hickey. 2001. Systematics of the Northern Andean Isoëtes karstenii complex. Am. Fern J. 91: 41-69.

Spencer, D.H.N. 1982. The zonation of plants in freshwater lakes. Adv. Ecol. Res. 12: 37-125.

Torres, B.E. 1985. Aspectos ecológicos del género Sphagnum en el Parque Nacional Natural de Chingaza. Bol. Dept. Biol. Fac. Cienc. Univ. Nacional 2: 57-67.

van Breemen, N. 1995. How Sphagnum bogs down other plants. Trends Ecol. Evol. 10: 270-275.

Vargas, O. 1996. Impacto del fuego y pastoreo sobre el medio ambiente páramo, p. 63-72. In P. Reyes Zambrano, J. Molano Barrero, C. Castaño Uribe \& O. Vargas Ríos (eds.). El páramo. Ecosistema de alta montaña. Fundación Ecosistemas Andinos ECOAN, Bogotá, Colombia.

Vargas, O. 1997. Un modelo de sucesión-regeneración de los páramos después de quemas. Caldasia 19: 331-345. 
Vargas, O. \& P. Pedraza. 2004. Parque Nacional Natural Chingaza. Universidad Nacional de Colombia, Colciencias, Convenio Unidad de Parques-Acueducto de Bogotá, Bogotá, Colombia.

Vargas, O., J. Premauer \& C.A. Cárdenas. 2002. Efecto del pastoreo sobre la estructura de la vegetación en un páramo húmedo de Colombia. Ecotrópicos 15: 33-48.

Vargas, O. \& D. Rivera. 1991. Comunidades vegetales del Parque Nacional Natural Chingaza: Sector I Río La Playa - Río Guatiquía (resultados preliminares). Cuadernos Divulgativos Univ. Javeriana 23: 1-74.

Vargas, O. \& S. Zuluaga. 1985. La vegetación del Páramo de Monserrate, p. 167-224. In H. Sturm \& J.O Rangel-Ch. (eds.). Ecología de los paramos andinos, una visión preliminar integrada. Bibl. J. J. Triana, 5. Instituto de Ciencias Naturales - Museo de Historia Natural, Facultad de Ciencias, Universidad Nacional de Colombia, Bogotá, Colombia.

Verweij, P.A. 1995. Spatial and temporal modelling of vegetation patterns: Burning and grazing in the páramo of los Nevados National Park, Colombia. Ph.D. Thesis, ITC Publication 30, Ámsterdam, Holanda.

Verweij, P.A. \& P.E. Budde. 1992 Burning and grazing gradients in páramo vegetation: Initial ordination analysis, p. 177-196. In H. Balslev \& J.L Luteyn (eds.). Páramo. An Andean ecosystem under human influence. Academic, Londres, Inglaterra.

Verweij, P.A. \& K. Kok. 1992. Effects of fire and grazing on Espeletia hartwegiana populations, p. 215-229. In H. Balslev \& J.L Luteyn (eds.). Páramo. An Andean ecosystem under human influence. Academic, Londres, Inglaterra.

Whittaker, H.R. 1970. The population structure of vegetation, p. 39-59. In R. Tüxen (ed.). Gesellschaftsmorphologie. Ber. Symp. Int. Ver. Vegetationskunde, Rinteln 1966. La Haya, Holanda.

Wiedermann, M.M., U. Gunnarsson, M.B. Nilsson \& N. Annika. 2009. Can small-scale experiments predict ecosystem responses? An example from peatlands. Oikos 118: 449-456. 


\section{APÉNDICE}

Lista taxonómica, código y clasificación biotipológica de los macrófitos acuáticos y semiacuáticos coleccionados en el PNN Chingaza

\section{APPENDIX}

Check list, codes, and life form classification of aquatic and wetland plants of the Chingaza Park

\section{Familia}

\section{Género/Especie}

\section{Código}

Nit-fle

SUM

* Nitella flexilis (L.) Agardh. var. flexilis f. colombiana Mora

2. Bryophyta

Marchantiaceae

Marchantia plicata Nees \& Mont.

Mar-pli

HEL-bri

Balantiopsaceae

Isotachis serrulata (Sw.) Gottsche

Amblystegiaceae

Drepanocladus exannulatus (Bruch. Schimp. \& W. Gümbel) Warnst.

Dre-exa

HEL-bri

Leptodictyum sp.

Scorpidium scorpioides (Hedw.) Limpr.

Bartramiaceae

Breutelia sp.

Hylocomiaceae

Pleurozium schreberi (Brid.) Mitt.

Lep-sp1

HEL-bri

Sphagnaceae

Sphagnum cuspidatum Ehrh. ex Hoffm.

Sphagnum spp.

Musgos indeterminados

3. Pteridophyta

Isoëtaceae

Isoëtes palmeri H.P. Fuchs

Isoëtes karstenii A. Braun

Equisetaceae

Equisetum bogotensis H. B. K.

Blechnaceae

Blechnum loxense Hook. ex Salomon

Salviniaceae

Azolla filiculoides Lam.

Azo-fil

ERR

4. Monocotyledoneas

Cyperaceae

* Carex acutata Boott.

Carex bonplandii Kunth

Carex conferto-spicata Boeck.

Bre-sp1

TER

Ple-sch

TER

Sph-cus

HEL-bri

Sph-spp

HEL-bri

Mus-spp

TER

Iso-pal

SUM

$+$
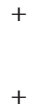

Carex pichinchensis H.B.K.

Carex teres Boott.

Cyperus bipartitus Torrey

Cyperus rufus H.B.K.

Eleocharis macrostachya Britt.

$\begin{array}{ll}\text { Car-acu } & \text { HEL-gra } \\ \text { Car-bon } & \text { HEL-gra } \\ \text { Car-con } & \\ \text { Car-pic } & \text { HEL-gra } \\ \text { Car-ter } & \text { HEL-gra } \\ \text { Cyp-bip } & \text { HEL-gra } \\ \text { Cyp-ruf } & \text { HEL-gra } \\ \text { Ele-mac } & \text { HEL-jun }\end{array}$


APÉNDICE (Continuación)

Lista taxonómica, código y clasificación biotipológica de los macrófitos acuáticos y semiacuáticos coleccionados en el PNN Chingaza

APPENDIX (Continued)

Check list, codes, and life form classification of aquatic and wetland plants of the Chingaza Park

\begin{tabular}{|c|c|c|}
\hline \multicolumn{3}{|c|}{ Familia } \\
\hline Género/Especie & Código & Biotipo \\
\hline Eleocharis stenocarpa Svenson & Ele-ste & HEL-jun \\
\hline Isolepis inundata $\mathrm{R} . \mathrm{Br}$. & Iso-inu & HEL-gra \\
\hline \multicolumn{3}{|l|}{ Eriocaulaceae } \\
\hline Paepalanthus columbiensis Ruhland & Pae-col & TER \\
\hline Paepalanthus karstenii Ruhl. & Pae-kar & TER \\
\hline \multicolumn{3}{|l|}{ Iridaceae } \\
\hline Sisyrinchium bogotense H.B.K. & Sis-bog & TER \\
\hline Sisyrinchium convolutum Nocca & Sis-con & TER \\
\hline \multicolumn{3}{|l|}{ Juncaceae } \\
\hline Juncus breviculmis Balslev & Jun-bre & HEL-jun \\
\hline Juncus cyperoides Lah. & Jun-cyp & HEL-gra \\
\hline Juncus ecuadoriensis Balslev & Jun-ecu & HEL-jun \\
\hline Juncus effusus L. & + & \\
\hline \multicolumn{3}{|l|}{ Poaceae } \\
\hline Alopecurus aequalis Sobol & Alo-aeq & HEL-gra \\
\hline Anthoxanthum odoratum $\mathrm{L}$. & Ant-odo & TER \\
\hline Calamagrostis effusa (H.B.K.) Steudel & Cal-eff & TER \\
\hline Calamagrostis planifolia (H.B.K.) Trin. & + & \\
\hline Chusquea tessellata Munro & Chu-tes & TER \\
\hline Festuca andicola Kunth & Fes-and & HEL-gra \\
\hline Muhlenbergia fastigiata (J. Presl) Henrard & Muh-fas & HEL-gra \\
\hline Paspalum hirtum H.B.K. & Pas-hir & TER \\
\hline * Paspalum cf. vaginatum $\mathrm{Sw}$. & Pas-vag & HEL-gra \\
\hline Phalaris angusta Nees ex Trin. & Pha-ang & HEL-gra \\
\hline Poa aпnиа $\mathrm{L}$. & Poa-ann & TER \\
\hline Potamogetonaceae & & \\
\hline Potamogeton illinoensis Morong. & Pot-ill & SUM \\
\hline Potamogeton paramoanus Haynes \& Holm-Nielsen & Pot-par & HOJ-flo \\
\hline Eudicotyledoneas & & \\
\hline Apiaceae & & \\
\hline Hydrocotyle bonplandii A. Rich. & Hyd-bon & HEL-her \\
\hline Hydrocotyle ranunculoides $\mathrm{L} . \mathrm{f}$. & Hyd-ran & HEL-her \\
\hline * Lilaeopsis macloviana (Gandoger) A.W. Hill & Lil-mac & HEL-jun \\
\hline Asteraceae & & \\
\hline Bidens triplinervia H.B.K. & Bid-tri & TER \\
\hline Espeletia grandiflora (Humb. \& Bonpl.) Cuatr. & + & \\
\hline Plagiocheilus solivaeformis DC. & Pla-sol & HEL-her \\
\hline Siegesbeckia jorullensis H.B.K. & + & \\
\hline Werneria pygmaea Gill. ex Hook. \& Arn. & + & \\
\hline Brassicaceae & & \\
\hline
\end{tabular}


APÉNDICE (Continuación)

Lista taxonómica, código y clasificación biotipológica de los macrófitos acuáticos y semiacuáticos coleccionados en el PNN Chingaza

APPENDIX (Continued)

Check list, codes, and life form classification of aquatic and wetland plants of the Chingaza Park

\begin{tabular}{|c|c|c|}
\hline \multicolumn{3}{|l|}{ Familia } \\
\hline Género/Especie & Código & Biotipo \\
\hline Cardamine bonariensis Pers. & Car-bon & HEL-her \\
\hline Cardamine killipii Schulz & + & \\
\hline \multicolumn{3}{|l|}{ Bromeliaceae } \\
\hline Puya santosii Cuatrec. & + & \\
\hline \multicolumn{3}{|l|}{ Callitrichaceae } \\
\hline Callitriche nubigena Fassett & Cal-nub & HOJ-flo \\
\hline \multicolumn{3}{|l|}{ Caryophyllaceae } \\
\hline Cerastium fontanum Baumg. & + & \\
\hline \multicolumn{3}{|l|}{ Crassulaceae } \\
\hline Crassula venezuelensis (Steyermark) Bywater \& Wickens & Cra-ven & SUM \\
\hline \multicolumn{3}{|l|}{ Elatinaceae } \\
\hline * Elatine paramoana Schmidt-Mumm \& Bernal & Ela-par & SUM \\
\hline \multicolumn{3}{|l|}{ Ericaceae } \\
\hline Pernettya prostrata (Cavan.) Sleumer var. myrsinoides (H.B.K.) Sleumer & Per-pro & TER \\
\hline \multicolumn{3}{|l|}{ Fabaceae } \\
\hline Trifolium repens $\mathrm{L}$. & Tri-rep & TER \\
\hline \multicolumn{3}{|l|}{ Gentianaceae } \\
\hline Halenia asclepiadea (H.B.K.) Don & Hal-asc & TER \\
\hline \multicolumn{3}{|l|}{ Geraniaceae } \\
\hline Geranium sibbaldiodes Benth. ssp. elongatum (Wedd.) Aedo & Ger-sib & TER \\
\hline \multicolumn{3}{|l|}{ Haloragaceae } \\
\hline Myriophyllum quitense H.B.K. & Myr-qui & SUM \\
\hline \multicolumn{3}{|l|}{ Hypericaceae } \\
\hline Hypericum juniperinum H.B.K. & Hyp-jun & TER \\
\hline \multicolumn{3}{|l|}{ Lamiaceae } \\
\hline Stachys elliptica Benth. & + & \\
\hline Lentibulariaceae & & \\
\hline Utricularia gibba $\mathrm{L}$. & + & \\
\hline Onagraceae & & \\
\hline Epilobium denticulatum Ruíz \& Pavón & Epi-den & HEL-her \\
\hline Polygonaceae & & \\
\hline Polygonum hydropiperoides Michx. & Pol-hyd & HEL-her \\
\hline Portulacaceae & & \\
\hline Montia biapiculata Lourteig. & Mon-bia & HEL-her \\
\hline Ranunculaceae & & \\
\hline Ranunculus flagelliformis J.E. Smith & Ran-fla & HEL-her \\
\hline Ranunculus limoselloides Turcz. & Ran-lim & HOJ-flo \\
\hline Ranunculus nubigenus H.B.K. & Ran-nub & HEL-her \\
\hline Rosaceae & & \\
\hline Lachemilla orbiculata (R. \& P.) Rydb. & Lac-orb & HEL-her \\
\hline
\end{tabular}


APÉNDICE (Continuación)

Lista taxonómica, código y clasificación biotipológica de los macrófitos acuáticos y semiacuáticos coleccionados en el PNN Chingaza

APPENDIX (Continued)

Check list, codes, and life form classification of aquatic and wetland plants of the Chingaza Park

\begin{tabular}{|c|c|c|}
\hline \multicolumn{3}{|c|}{ Familia } \\
\hline Género/Especie & Código & Biotipo \\
\hline Lachemilla sp. & Lac-sp1 & TER \\
\hline \multicolumn{3}{|l|}{ Rubiaceae } \\
\hline Arcytophyllum muticum (Wedd.) Stand. & Arc-mut & TER \\
\hline Nertera granadensis (Mutis ex L. f.) Druce & Ner-gra & TER \\
\hline Galium ascendens Willd. ex Spreng. & Gal-asc & HEL-her \\
\hline \multicolumn{3}{|l|}{ Scrophulariaceae } \\
\hline Aragoa abietina H.B.K. & Ara-abi & TER \\
\hline Bartsia sp. & + & \\
\hline Calceolaria mexicana Benth. subsp. mexicana & + & \\
\hline Gratiola bogotensis Cortés & Gra-bog & HEL-her \\
\hline Limosella australis $\mathrm{R} . \mathrm{Br}$. & Lim-aus & HEL-jun \\
\hline Mimulus glabratus H.B.K. & Mim-gla & HEL-her \\
\hline Veronica serpyllifolia $\mathrm{L}$. & Ver-ser & TER \\
\hline \multicolumn{3}{|l|}{ Valerianaceae } \\
\hline Valeriana sp. & Val-sp1 & TER \\
\hline
\end{tabular}

Especies marcadas con un asterisco $\left(^{*}\right)$ representan un primer registro para el Parque, las que no tienen código (+) se encuentran excluidas del análisis de las comunidades por su escasa representación.

Species with an asterisk (*) are representing a first record for the Park. Rare species (+) are not coded and where excluded from data analysis. 